Z Rheumatol 2021 $\cdot 80: 670-687$ https://doi.org/10.1007/s00393-021-01028-w Angenommen: 29. April 2021 Online publiziert: 6 . August 2021 (c) Springer Medizin Verlag $\mathrm{GmbH}$, ein Teil von Springer Nature 2021, korrigierte Publikation 2022

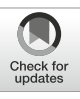

\section{Zusatzmaterial online}

Zusätzliche Informationen sind in der Online-Version dieses Artikels (https:// doi.org/10.1007/s00393-021-01028-w) enthalten.

\section{Einleitung}

Glukokortikoide (engl. „glucocorticoids", Abk.: GC) sind aufgrund ihrer potenten antiinflammatorischen und immunsuppressiven Eigenschaften von erheblicher therapeutischer Bedeutung und werden vielfach bei Patienten mit chronisch entzündlichen Erkrankungen

Die englische Version dieses Beitrags ist unter https://doi.org/10.1007/s00393-021-01025-z zufinden.

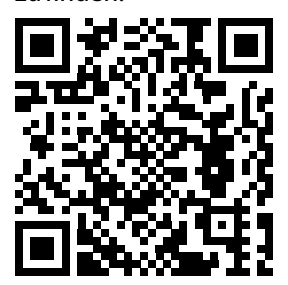

QR-Code scannen \&Beitrag online lesen

Jan Leipe ${ }^{1,2}$ für die Kommission Pharmakotherapie der Deutschen Gesellschaft für Rheumatologie e. V. (DGRh) · Julia U. Holle für die Kommission Pharmakotherapie der Deutschen Gesellschaft für Rheumatologie e. V. (DGRh) . Christiane Weseloh ${ }^{4}$ für die Kommission Pharmakotherapie der Deutschen Gesellschaft für Rheumatologie e. V. (DGRh) · Alexander Pfeil ${ }^{5} \cdot$ Klaus Krüger $^{6}$ für die Kommission Pharmakotherapie der Deutschen Gesellschaft für Rheumatologie e.V. (DGRh)

'Sektion Rheumatologie, Medizinische Klinik V, Universitätskrankenhaus Mannheim, Universitätsklinikum Mannheim, Mannheim, Deutschland; ' ${ }^{2}$ Sektion Rheumatologie und Klinische Immunologie, Medizinische Klinik IV, Ludwig-Maximilians-Universität München, München, Deutschland; ${ }^{3}$ Rheumazentrum Schleswig-Holstein Mitte, Neumünster, Deutschland; ${ }^{4}$ Deutsche Gesellschaft für Rheumatologie, Berlin, Deutschland; ${ }^{5}$ Klinik für Innere Medizin III, Funktionsbereich Rheumatologie, Universitätsklinikum Jena, Jena, Deutschland; ${ }^{6}$ Rheumatologisches Praxiszentrum München, München, Deutschland

\title{
Empfehlungen der Deutschen Gesellschaft für Rheumatologie zum Management der Glukokortikoid-induzierten Osteoporose
}

eingesetzt. In der Rheumatologie werden GC seit mehr als 70 Jahren genutzt und zählen zu den häufigsten verordneten Medikamenten bei entzündlich rheumatischen Erkrankungen (ERE). Eine Langzeit-GC-Therapie wird als eine Behandlung mit GC über $\geq 3$ Monaten definiert [7], wobei in der aktuellen Literatur keine einheitliche GC-Mindestdosis (früher teilweise mit $7,5 \mathrm{mg}$ Prednisolonäquivalent pro Tag angegeben) definiert ist. Die fehlende Einheitlichkeit basiert auf den Erkenntnissen, dass eine Langzeittherapie mit GC auch in niedrigeren Dosen (bis zu 2,5 mg Prednisolonäquivalent) bereits mit einem Knochenmineraldichte (engl. „, bone mineral density", Abk.: BMD)-Verlust assoziiert ist [10, 56, 91].

\section{Epidemiologie der Glukokortikoid- induzierten Osteoporose}

In der Literatur wird geschätzt, dass ca. 0,5-1,0\% der Bevölkerung in westlichen Ländern langfristig mit GC behandelt werden [5]. Die langfristige Anwendung von GC ist mit erheblichen Nebenwir-

\begin{tabular}{|ll}
\hline \multicolumn{2}{|l}{ Abkürzungen } \\
\hline$A C R$ & American College of Rheumatology \\
\hline$B M D$ & Knochenmineraldichte \\
\hline$D E N$ & Denosumab \\
\hline$D V O$ & Dachverband Osteologie \\
\hline$D X A$ & Dual-Energy-X-ray-Absorptiometrie \\
\hline FRAX & Fracture Risk Assessment Tool \\
\hline$G C$ & Glukokortikoide \\
\hline$G F R$ & Glomeruläre Filtrationsrate \\
\hline$G I O P$ & $\begin{array}{l}\text { Glukokortikoid-induzierte Osteopo- } \\
\text { rose }\end{array}$ \\
\hline$K I$ & Konfidenzintervall \\
\hline$L W S$ & Lendenwirbelsäule \\
\hline$n . S$. & Nicht signifikant \\
\hline$N N T$ & Number needed to treat \\
\hline$R C T$ & Randomized Controlled Trial \\
\hline$S L R$ & Systematische Literaturrecherche \\
\hline$T E R$ & Teriparatid \\
\hline
\end{tabular}


kungen assoziiert, wobei die mit einem BMD-Verlust einhergehende Osteoporose und Frakturen die häufigsten, zumindest teilweise vermeidbaren sowie schweren Nebenwirkungen einer GC-Therapie darstellen [70, 81, 88]. In diesem Zusammenhang ist die Glukokortikoid-induzierte Osteoporose (GIOP) als häufigste Ursache einer sekundären Osteoporose zu nennen. Bei mehr als $10 \%$ der Patienten unter einer Langzeit-GC-Therapie wird eine osteoporotische Fraktur diagnostiziert, und bei ca. 30-40\% der Patienten finden sich radiologische Hinweise auf Frakturen, wobei insgesamt nur ein Drittel der Patienten über Fraktursymptome berichtet $[12,59]$. Es wird vermutet, dass dieser geringe Anteil an symptomatischen Patienten durch die analgetische Wirkung der GC bedingt ist [75]. Nicht zuletzt ist aufgrund der mehrheitlich asymptomatischen Verläufe bei der GIOP ein aktives, auf die Prophylaxe von Frakturen ausgerichtetes Osteoporosemanagement erforderlich. Jedoch zeigte eine kanadische Studie, dass nur bei $25 \%$ der Patienten, bei denen eine Langzeit-GC-Therapie initiiert wurde, ein Osteoporosemanagement (z.B. Risikoevaluation, Basisdiagnostik, BMD-Messung oder Osteoporosetherapie) durchgeführt wurde [58].

\section{Entstehung GIOP und Risiko von Frakturen unter Glukokortikoiden}

Die zugrunde liegenden pathophysiologischen Mechanismen der GIOP führen $\mathrm{zu}$ einer erhöhten Knochenfragilität durch eine verminderte Knochenneubildung verbunden mit erhöhter Knochenresorption. Eine erhöhte Knochenresorption wird $\mathrm{v}$. a. in den frühen Phasen der GC-Behandlung beobachtet, während die verminderte Knochenneubildung nach mehreren Monaten der Behandlung deutlich wird [4].

Von Beginn an ist eine GC-Therapie bereits mit einem Anstieg des Frakturrisikos assoziiert [67].

Mit der Initiierung einer GC-Therapie beträgt die jährliche Inzidenz vertebraler und nichtvertebraler Frakturen 5,1\% bzw. 2,5\% und bei Patienten, die bereits GC seit mehr als 6 Monaten erhielten, jeweils 3,2 und 3,0\% [10]. Im Ver- gleich dazu lag die jährliche Inzidenz für vertebrale Frakturen (1,1\% Frauen und 0,6\% Männer) [32] und nichtvertebrale Frakturen (Frauen: 0,1-3,6\%; Männer: 0,1-2,0\%) in der Allgemeinbevölkerung deutlich niedriger [41]. Die höchste Frakturinzidenz im Rahmen der Therapie mit GC besteht im ersten Behandlungsjahr und kann durch eine starke GC-Wirkung auf Knochenumbauprozesse erklärt werden. Der höchste Knochenabbau wird innerhalb der ersten 3 bis 6 Monate nach der Einleitung einer GC-Therapie beobachtet, und der Verlust an der BMD am Ende des ersten Jahres beträgt bis zu $12 \%$. Im weiteren Verlauf tritt ein langsamerer BMD-Verlust (2-3\% pro Jahr) bei Langzeit-GCTherapie (>5 mg je Tag) auf $[10,56,91]$. Als wesentliche Faktoren für das Frakturrisiko sind die GC-Tagesdosis und die Anwendungsdauer (kumulative GCDosis) zu nennen. Dementsprechend weisen Patienten mit einer hohen GCDosis (Tagesdosis $\geq 15 \mathrm{mg}$ Prednisonäquivalent und/oder kumulative Dosis $\geq 1 \mathrm{~g}$ ) ein um ein Vielfaches erhöhtes Risiko (Odds Ratio 2,9) für Frakturen im Vergleich zu Patienten mit einer geringeren Tages- bzw. Kumulativdosis auf [9]. Das Frakturrisiko steigt vermutlich schon ab GC-Dosen oberhalb von 2,5 mg Prednisolonäquivalent pro Tag an [22, 90]. Das relative adjustierte Frakturrisiko betrug in einer britischen Datenbankstudie für proximale Femurfrakturen 0,99 (<2,5 mg Prednisolonäquivalent), 1,77 (2,5-7,5 mg Prednisolonäquivalent) sowie 2,27 (7,5 mg Prednisolonäquivalent). Für vertebrale Frakturen konnte ebenfalls eine ähnliche Dosisabhängigkeit zwischen dem relativen Risiko für das Auftreten von vertebralen Frakturen und der GC-Dosis ermittelt werden: 1,55 (<2,5 mg), 2,59 (2,5-7,5 mg) und 5,18 ( $\geq 7,5 \mathrm{mg}$ Prednisolonäquivalent) [90].

Eine GC-Therapie beeinflusst sämtliche Knochenkompartimente, wobei der trabekuläre Knochen (Abnahme in ersten 20 Wochen: $-8,2 \%, p<0,05)$ deutlich stärker als der kortikale Knochen (Abnahme in ersten 20 Wochen: $-2,1 \%$, $p=$ n. s.) betroffen ist $[9,15,89,90]$. Daher treten Frakturen am häufigsten an Knochenstrukturen mit einem hohen Anteil von trabekulären Knochen auf (z. B. Len- denwirbelsäule und proximales Femur) $[12,88]$.

Die negativen Effekte einer GC-Behandlung auf den Knochen sind jedoch ein potenziell reversibler Risikofaktor für die GIOP. Die Beendigung einer GCBehandlung ist mit einem potenziellen Anstieg der BMD und einer Erniedrigung des Frakturrisikos verbunden [39, $48,90]$. Zudem ist eine kurzzeitige hoch dosierte GC-Therapie nur mit einem geringen Anstieg des Frakturrisikos assoziiert [29]. Interessanterweise ist das Risiko einer GC-induzierten Fraktur bereits erhöht, bevor es zu einer signifikanten Reduktion der BMD kommt; dieses erhöhte Frakturrisiko ist vermutlich Ausdruck einer verminderten Knochenqualität unter einer GC-Therapie [88]. Daraus resultiert, dass die alleinige BMD-Messung zur Beurteilung des Frakturrisikos unter einer GC-Therapie nicht ausreichend ist und eine Risikoevaluation bezüglich des Frakturrisikos durchzuführen ist. Als weitere Risikofaktoren für das Auftreten einer GIOP-assoziierten Fraktur sind das Alter, Geschlecht, Komorbiditäten, Nikotinkonsum und die Begleitmedikation zu nennen, welche die Entscheidung bezüglich einer therapeutischen Intervention beeinflussen [1].

\section{Einschätzung des Frakturrisikos und Evaluation der Osteoporose hinsichtlich Therapiebedürftigkeit}

Um das Frakturrisiko einschätzen zu können, wurden verschiedene Risikokalkulatoren implementiert, wobei die GC-Dosis in einigen, aber nicht allen Kalkulatoren Eingang gefunden hat. Die meisten Risikokalkulatoren stratifizieren die GC-Dosis in 2 Kategorien: Niedrigdosisbereich (Prednisolonäquivalent: $\leq 7,5 \mathrm{mg} / \mathrm{Tag}$ ) und Hochdosisbereich (Prednisolonäquivalent: $>7,5 \mathrm{mg} / \mathrm{Tag}$ ). Die Einteilung basiert auf Daten aus klinischen und epidemiologischen Studien, welche ein steigendes Frakturrisiko mit höheren GC-Tagesdosen belegen [29, 44]. Diese Risikorechner unterschätzen jedoch möglicherweise das Frakturrisiko bei Patienten mit einer zeitlich prolongierten GC-Behandlung (z.B. Polymyalgia rheumatica und Riesenzellarteriitis) [29, 36]. Ein international 
Z Rheumatol 2021 · 80:670-687 https://doi.org/10.1007/s00393-021-01028-w

(c) Springer Medizin Verlag GmbH, ein Teil von Springer Nature 2021

J. Leipe für die Kommission Pharmakotherapie der Deutschen Gesellschaft für Rheumatologie e. V. (DGRh) · J. U. Holle für die Kommission Pharmakotherapie der Deutschen Gesellschaft für Rheumatologie e. V. (DGRh) · C. Weseloh für die Kommission Pharmakotherapie der Deutschen Gesellschaft für Rheumatologie e. V. (DGRh) · A. Pfeil · K. Krüger für die Kommission Pharmakotherapie der Deutschen Gesellschaft für Rheumatologie e. V. (DGRh)

\section{Empfehlungen der Deutschen Gesellschaft für Rheumatologie zum Management der Glukokortikoid- induzierten Osteoporose}

\section{Zusammenfassung}

Hintergrund. Glukokortikoide sind von erheblicher therapeutischer Bedeutung bei der Behandlung entzündlicher Erkrankungen, jedoch insbesondere bei langfristiger Anwendung mit Knochenmineraldichteverlust, Osteoporose und Frakturen assoziiert.

Ziel dieser Arbeit. Es sollten Empfehlungen zum Management der Glukokortikoid-induzierten Osteoporose (GIOP) bei erwachsenen Patienten unter Langzeit-Glukokortikoid(GC)Therapie entwickelt werden.

Methodik. Es wurde eine systematische Literatursuche (SLR) durchgeführt, um die Evidenz für Präventions- und Behandlungsoptionen der GIOP zusammenzufassen. Mithilfe zuvor definierter Fragestellungen wurden, basierend auf SLR und dem Evidenzgrad,
Empfehlungen in einem strukturierten Gruppenkonsensprozess erarbeitet. Ergebnisse. Die Empfehlungen umfassen zunächst die Supplementation mit Kalzium und Vitamin D bei Erwachsenen unter Langzeit-GC-Therapie. Wenn eine spezifische osteologische Therapie angezeigt ist, empfehlen wir als Erstlinientherapie Bisphosphonate oder Denosumab. Bei hohem Frakturrisiko empfehlen wir Teriparatid als primäre spezifische osteologische Therapie. Bei hochgradiger Niereninsuffizienz soll Denosumab (nach Ausschluss renaler Osteopathie) eingesetzt werden und bei Schwangerschaft keine spezifische osteologische Therapie erfolgen. Für Patienten, die das Therapieziel nicht erreicht haben, sollte die Umstellung auf eine andere Klasse spezifischer osteologischer Medikamente erfolgen. Wir empfehlen eine Reevaluation nach einer Therapiedauer von 3 bis 5 Jahren oder nach Beendigung der Langzeit-GC-Therapie.

Fazit. Diese Arbeit soll evidenz- und konsensbasierte Empfehlungen für ein bestmögliches Management der GIOP in Deutschland geben und bei Behandlungsentscheidungen unterstützen.

Schlüsselwörter

Rheumatische Erkrankungen · Knochenmineraldichteverlust - Osteoporose . Glukokortikoide · Langzeittherapie

\section{German Society of Rheumatology Recommendations for the management of glucocorticoid-induced Osteoporosis. German version}

\section{Abstract}

Background. Glucocorticoids are of substantial therapeutic importance in the treatment of inflammatory diseases, but are also associated with bone mineral density loss, osteoporosis, and fractures, especially with long-term use. Objective. To develop recommendations for the management of glucocorticoid-induced osteoporosis (GIOP) in adult patients on longterm glucocorticoid (GC) treatment.

Methods. A systematic literature search (SLR) was conducted to synthesize the evidence for GIOP prevention and treatment options. Recommendations were developed based on SLR/level of evidence and by previously defined questions and in a structured group consensus process.

Results. Recommendations include supplementation with calcium and vitamin $D$ under long-term GC therapy in adults. If specific osteologic treatment is indicated, we recommend bisphosphonates or denosumab as first-line treatment. If fracture risk is high, we recommend teriparatide as primary specific osteologic treatment. Denosumab should be used in cases of severe renal insufficiency, and specific osteologic treatment should not be given in pregnancy. For patients who have not reached the treatment goal, a switch to another class of specific osteologic drugs should be performed. We recommend reevaluation after a treatment duration of 3-5 years or after termination of long-term GC treatment.

Conclusion. This work aims to provide evidence-based and consensus-based recommendations for the best possible management of GIOP in Germany and to support treatment decisions.

\section{Keywords}

Rheumatic disease - Bone mineral density loss · Osteoporosis · Glucocorticoids · Longterm treatment häufig verwendeter Risikokalkulator zur Bestimmung des Frakturrisikos und zur Abschätzung einer möglichen Therapieindikation stellt das Fracture Risk Assessment Tool (FRAX) dar. Zu den im FRAX enthaltenen Parametern werden als additive Risikofaktoren für GC-induzierte Frakturen eine geringe Knochenfestigkeit zu Beginn der GC-Behandlung sowie die Geschwindigkeit des BMD-Verlusts während der Behandlung vorgeschla- gen [20]. Im deutschsprachigen Raum wird die Leitlinie des Dachverbandes Osteologie (DVO) zur Beurteilung des Frakturrisikos und einer Therapienotwendigkeit angewendet [1].

Die DVO-Leitlinie (2017) empfiehlt bei einer bestehenden oder geplanten GC-Therapie ( $\geq 2,5 \mathrm{mg}$ Prednisolonäquivalent über mehr als 3 Monate) bei postmenopausalen Frauen und Männern ab dem 50. Lebensjahr eine Basisdiagnos- tik [1]. Bei jüngeren Patienten (Frauen: $<50$ Jahre und Männer <60 Jahre) sollte entsprechend der DVO-Leitlinie als Einzelfallentscheidung bereits zu einem früheren Zeitpunkt eine Basisdiagnostik initiiert werden. Die Basisdiagnostik umfasst die folgenden Schritte:

1. Anamneseerhebung in Bezug auf das Frakturrisiko (z. B. Sturzrisikoevaluation, aufgetretene Frakturen [Wirbelkörperfrakturen], Frakturen 


\section{Infobox 1 Osteoporose \\ Definition: Die Osteoporose ist eine systemische Skeletterkrankung, die durch eine niedrige Knochenmasse und eine mikroarchitektonische Verschlechterung des Knochengewebes charakterisiert ist, mit einem konsekutiven Anstieg der Knochenfragilität und der Neigung zu Frakturen. Sind bereits Frakturen als Folge der Osteoporose aufgetreten, liegt eine manifeste Osteoporose vor (http://dv-osteologie.org/ osteoporose-leitlinien) [1]. \\ Die WHO-Definition einer Osteoporose basiert auf einer DXA-Knochenmineraldich- temessung, bei der eine Erniedrigung des T-Scores $\leq-2,5$ Standardabweichungen als die Osteoporose festgelegt wird. Als Osteopenie wird eine Erniedrigung des T-Scores zwischen $-1,0$ Standardabweichung und $-2,5$ Standardabweichung definiert [13].}

in der Familienanamnese [Femurfraktur bei Eltern]) und Beurteilung der Muskelkraft sowie Koordination,

2. Erhebung des Basislabors: Serumkalzium, Serumphosphat, alkalische Phosphatase, Gamma-GT, KreatininClearance, Blutsenkungsgeschwindigkeit/C-reaktives Protein, Blutbild, Thyreoidea-stimulierendes Hormon, Serumeiweißelektrophorese (Immunfixation optional) (ggf.: 25Hydroxy-Vitamin $\mathrm{D}_{3}$ und Knochenresorptionsparameter in Einzelfällen, Testosteron bei Männern),

3. Knochenmineraldichtemessung mittels des Standardverfahrens Dual-Energy-X-ray-Absorptiometrie (DXA).

4. Bei klinischen Hinweisen auf Wirbelkörperfrakturen sollte eine Bildgebung (Röntgenuntersuchung bzw. eine alternative Bildgebungsmethode) erfolgen.

Bezüglich BMD-Messung und Definition der Osteoporose gilt Folgendes: In der DVO-Leitlinie wird als Standardverfahren eine DXA-Messung an der Lendenwirbelsäule (LWS) und am proximalen Femur definiert [1].

Die WHO-Definition der Osteoporose basiert auf den T-Scores der DXAMethode und ist mit einer Erniedrigung des T-Scores $\leq-2,5$ Standardabweichungen definiert (WHO-Definition [13]).
Der T-Score (T-Wert) gibt die Abweichung des BMD-Messwertes des Patienten vom Durchschnittswert der Spitzen-BMD knochengesunder Menschen an [54]. Der T-Score wird an der LWS und am proximalen Femur bestimmt. An der LWS wird der mittlere T-Score derjenigen Wirbel (Lendenwirbelkörper 1 bis 4) ermittelt, an denen eine auswertbare Messung möglich ist, wobei mindestens 2 Wirbelkörper beurteilbar sein müssen. Am proximalen Femur sind der T-Score der Gesamtfemurregion (engl.: „total hip“) und der T-Score am Femurhals als Messorte zu bevorzugen. Es wird der niedrigste der 3 T-Scores zur Beurteilung herangezogen. Weitere Methoden zur BMD-Messung (quantitativer Ultraschall und quantitative Computertomographie, Trabecular Bone Score) sollten gemäß der DVO-Leitlinie (2017) nur in Ausnahmefällen, wenn eine DXA-Knochenmineraldichtemessung nicht möglich oder verwertbar ist, zur Anwendung kommen. Als Ursache hierfür ist anzuführen, dass für die alternativen Messmethoden keine validierten Referenzkohorten zur Verfügung stehen, und somit sind die herstellerseitig angegebenen T-Scores der Methoden nicht auf die T-Scores der DXA-Technik übertragbar.

Weiterführend wird im englischsprachigen Raum die Indikation zur DXAMessung meist unabhängig vom Alter mit Beginn einer Langzeit-GC-Therapie gestellt [18]. Eine Wiederholung der BMD-Messung sollte bei einer Änderung der Gesamtsituation in Bezug auf eine osteologische Therapieänderung erfolgen und ist in speziellen Situationen schon nach 12 bis 24 Monaten als sinnvoll anzusehen (http://dv-osteologie. org/osteoporose-leitlinien).

\section{Empfehlungen zum Management Glukokortikoid-induzierter Osteoporose}

Trotz zunehmender Evidenz bezüglich der GIOP und trotz Verfügbarkeit wirksamer osteologischer Therapien erhalten immer noch viele Patienten mit einer Langzeit-GC-Therapie keine geeignete Risikobeurteilung bzw. Diagnostik und keine entsprechende osteologische Behandlung. Oft werden erst nach Ent- wicklung einer GIOP oder nach dem Auftreten einer Fraktur osteologische Therapien initiiert $[33,80]$.

Nachdem die Deutsche Gesellschaft für Rheumatologie e. V. (DGRh), das Management der GIOP als ein wichtiges Thema identifizierte, wurden die vorliegenden Behandlungsempfehlungen entwickelt, um Hilfestellung für spezielle Belange in der Betreuung von Patienten mit Langzeit-GC-Therapie zu geben. Ziel der hier vorgestellten Empfehlungen ist es, eine evidenz- und konsensbasierte Empfehlung für ein bestmögliches Management der GIOP in Deutschland nach dem aktuellen Stand der Wissenschaft zu geben. Geltungsbereich der Empfehlung sind erwachsene Frauen und Männer mit einer GIOP.

In Bezug auf die analysierten Studien ist anzumerken, dass keine einheitliche Definition einer GIOP vorliegt. Weiterführend bestehen in den Studien deutliche Unterschiede hinsichtlich der GCTherapiedauer und der GC-Dosis. Hinsichtlich der Studienendpunkte wurde in den älteren Studien die Änderung der BMD als Endpunkt gesetzt, wohingegen in den neueren Studien die Reduktion der Frakturrate als Endpunkt definiert wurde. Dies führt ebenfalls zu keiner einheitlichen Betrachtung der Studienergebnisse.

In der DVO-Leitlinie (2017) werden 2 Ausgangspunkte zur Einleitung einer Osteoporosetherapie definiert: (I) Patienten, die mit $\geq 7,5 \mathrm{mg}$ Prednisolonäquivalent länger als 3 Monate behandelt werden und einen DXA-T-Score $\leq-1,5$ an der LWS aufweisen, sowie (II) eine Therapieeinleitung entsprechend dem Risikoprofil (Faktoren: Geschlecht, Lebensalter, DXA-Knochendichte und weitere Risikofaktoren) [1].

\section{Methoden}

Die Empfehlungen wurden in einem strukturierten Konsensprozess erarbeitet. Nach einer ersten konstituierenden Konferenz wurde dieser Prozess nach Definition der Arbeitsschritte in 5 weiteren Treffen fortgeführt. Zunächst erfolgte eine systematische Literaturrecherche (SLR). 


\section{Systematische Literaturrecherche}

Die SLR erfolgte in Anlehnung an die 2017 aktualisierte Leitlinie des American College of Rheumatology (ACR) $[19,20]$. Die Recherche wurde in den Datenbanken Medline (PubMed) sowie Cochrane Library vorgenommen und umfasste den Zeitraum 01.04.2016 bis 31.03.2020 (Suchstrategien s. Supplement 1). Die SLR ergab nach Dublettenabgleich 2958 Treffer (Abstracts). Die Daten wurden von der wissenschaftlichen Mitarbeiterin der DGRh (C.W.) in ein Abstractverwaltungsprogramm (Rayyan) hochgeladen und dort weiter selektiert (Titel/Abstract; s. Supplement). Die Vorselektion führte zum Ausschluss von 1338 Abstracts (duplicate, wrong drug, wrong intervention, wrong population type, wrong study design). Es wurden 41 Referenzen eingeschlossen. Bei 1730 Referenzen blieb die Entscheidung offen. Die Rechercheergebnisse (Abstracts) wurden allen Mitgliedern der Arbeitsgruppe in Rayyan zur weiteren Prüfung und Auswahl zur Verfügung gestellt. Die Mitglieder der Arbeitsgruppe selektierten aus der Vorauswahl 192 Abstracts. In einem weiteren Schritt wurde von den Mitgliedern der Arbeitsgruppe detaillierter selektiert.

Als Selektionskriterien hatten die Mitglieder der Arbeitsgruppe zuvor folgende Einschlusskriterien definiert:

- Population: erwachsene Frauen und Männer mit einer GIOP

- Publikationen: RCTs ( $n \geq 50)$, systematische Reviews, Metaanalysen

- Sprache: deutsch und englisch

Ausschlusskriterien:

- Population: Alter $\leq 18$ Jahre

- Publikationen: Kasuistiken und Kongressabstracts

Nach Daten zu folgenden Substanzen wurde gezielt gesucht:

- Zugelassene Wirkstoffe als antiresorptive Therapie: Bisphosphonate (Alendronat, Ibandronat, Risedronat, Zoledronat), Denosumab

- Zugelassene Wirkstoffe als osteoanabole Therapie: Teriparatid

- Supplementation: Vitamin $\mathrm{D}_{3}$ oder Kalzium, Vitamin $\mathrm{D}_{3}$ und Kalzium
Der ACR-Leitlinie von 2017 [19, 20] wurden im Rahmen der Handsuche weitere Quellen $(n=55)$ entnommen. Die weitere Handsuche ergab 36 Treffer.

Für das einleitende Kapitel zur Epidemiologie wurde keine SLR durchgeführt. Hier wurden über die Handsuche entsprechende Publikationen identifiziert. Nach Abschluss dieser Selektionsarbeiten wurden 102 Referenzen für die weitere Bewertung evaluiert und die entsprechenden Volltexte studiert.

\section{Evidenztabellen}

Die Mitglieder der Arbeitsgruppe waren beauftragt, anhand der Volltexte die für ihre Fragestellungen (s. unten) jeweils relevanten Studien für die weitere Evidenzbewertung herauszusuchen. Hauptkriterium für die Auswahl war die inhaltliche Relevanz für die jeweilige Fragestellung. Dabei wurden v. a. randomisiert kontrollierte Studien (RCTs) ausgewählt, insgesamt gingen 76 Publikationen in die Evidenzbewertung ein.

Folgende detaillierte Fragestellungen wurden von den Mitgliedern der Arbeitsgruppe zuvor festgelegt und für die Bearbeitung untereinander aufgeteilt:

1. Was für einen/keinen Nutzen hat die Supplementation mit Kalzium bei GIOP?

2. Was für einen/keinen Nutzen hat die Supplementation mit Vitamin $\mathrm{D}_{3}$ bei GIOP?

3. Was für einen/keinen Nutzen hat die Supplementation mit Vitamin $\mathrm{D}_{3}$ und Kalzium bei GIOP?

4. Was für einen/keinen Nutzen zeigt ein orales Bisphosphonat bei GIOP?

5. Was für einen/keinen Nutzen zeigt ein intravenöses Bisphosphonat bei GIOP?

6. Ist eine orale BisphosphonatTherapie einer intravenösen Bisphosphonat-Therapie bei GIOP überlegen?

7. Was für einen/keinen Nutzen zeigt Teriparatid bei GIOP?

8. Ist eine osteoanabole Therapie mit Teriparatid einer BisphosphonatTherapie bei GIOP überlegen?

9. Ist eine osteoanabole Therapie mit Teriparatid einer DenosumabTherapie bei GIOP überlegen?
10. Was für einen/keinen Nutzen zeigt Denosumab bei GIOP?

11. Ist eine Therapie mit Denosumab einer Bisphosphonat-Therapie bei GIOP überlegen?

12. Welche antiresorptiven und osteoanabolen Therapieverfahren können bei einer chronischen Niereninsuffizienz bei GIOP eingesetzt werden?

Das Erstellen der Evidenztabellen (s. Supplement) wurde von den Mitgliedern der Arbeitsgruppe vorgenommen. Dazu wurde die Klassifikation des Oxford Center of Evidence-based Medicine-Levels of Evidence von 2009 herangezogen (www.cebm.net).

Die Empfehlungen wurden in mehreren Telefon- bzw. Videokonferenzen (01.10.2019, 29.04.2020, 14.05.2020, 09.06.2020, 09.07.2020, 26.01.2021) erarbeitet.

\section{Externe Begutachtung und Verabschiedung}

Die Empfehlungen wurden vom Vorstand der DGRh begutachtet und am 07.04.2021 verabschiedet.

Es wurden nur Veröffentlichungen eingeschlossen, die auf randomisierten klinischen Studien bei Erwachsenen basierten, die Literatur umfasste die oben genannten zugelassenen Therapien in Deutschland für die Behandlung der Osteoporose bis zum Zeitpunkt 06/2020.

\section{Ergebnisse}

\section{Kalzium und Vitamin D}

Es existieren nur wenige randomisierte Studien, welche den Nutzen von Kalzium, Vitamin D und deren Kombination untersuchten.

Bezüglich des Effektes von Vitamin D wurde in einer doppelblinden, placebokontrollierten Studie bei 14 GC-therapierten Patienten demonstriert, dass Vitamin D im Vergleich zu Placebo in osteologischen Knochenbiopsien zu einem Anstieg des trabekulären Knochenvolumens und einer Abnahme der aktiven Resorption nach 6 Monaten führt (jeweils $p<0,05)$ [18]. Hinsichtlich des Ef- 
Hier steht eine Anzeige.

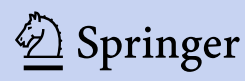


fekts der Kombination von Kalzium und Vitamin D zeigte sich in einer weiteren placebokontrollierten Studie mit 62 GCbehandelten Patienten, dass der BMDAbfall unter der Kombination Kalzium plus Vitamin $\mathrm{D}_{3}$ im Vergleich zu Placebo nach 12 Monaten geringer war, wenn auch nicht signifikant (12 Monate: $-2,6 \%$ vs. $-4,1 \%$; 24 Monate: $-3,7 \%$ vs. $-3,8 \%$ und 36 Monate: $-2,2 \%$ vs. $-1,5 \%$ ) [2]. ${ }^{1}$

Bezüglich der Effekte einer Kalziumbzw. Vitamin-D-Monotherapie und der Kombination von Kalzium und Vitamin $\mathrm{D}_{3}$ ergab eine dreiarmige „openlabel“ randomisiert kontrollierte Studie mit 66 GC-behandelten Patienten, dass die Behandlung mit Vitamin D (als Monotherapie) und Vitamin D plus Kalzium $\left(0,81 \mathrm{~g} / \mathrm{cm}^{2}\right.$ und $\left.0,82 \mathrm{~g} / \mathrm{cm}^{2}\right)$ mit signifikant höheren Knochenmineraldichtewerten an der LWS im Vergleich zur Kalziummonotherapie nach 24 Wochen assoziiert war $\left(0,62 \mathrm{~g} / \mathrm{cm}^{2}, p<0,05\right.$ jeweils) [23]. Auch in dieser Studie war die BMD in allen 3 Armen rückläufig. In keinem RCT wurde bei einer GIOP die Frakturreduktion dezidiert untersucht.

Insgesamt deuten die Daten, insbesondere auch unter Berücksichtigung von randomisierten placebokontrollierten Osteoporosestudien [19, 43, 76], dennoch darauf hin, dass die Kombination von Kalzium und Vitamin D im Vergleich zu Placebo einen positiven Effekt auf die BMD zeigt. Daher sollte bei Patienten unter einer Langzeit-GCTherapie die Zufuhr einer ausreichenden Menge an Kalzium (1000 mg/Tag) und Vitamin D (800 IE/Tag) sichergestellt werden.

Obgleich das Evidenzniveau insgesamt als gering zu bewerten ist, empfehlen wir in Übereinstimmung mit der DVO-Leitlinie (2017) (Prophylaxe, Diagnostik und Therapie der Osteoporose bei postmenopausalen Frauen und bei Männern) [1] und des American College of Rheumatology [19, 20] eine tägliche Zufuhr von $1000 \mathrm{mg} / \mathrm{Tag}$ Kalzium und 800 IE/Tag Vitamin D (25-Hydroxy-Vitamin-D-Serum-Zielkonzentration im

\footnotetext{
1 Standardabweichung und Konfidenzintervalle sind zur besseren Lesbarkeit des Manuskripttextes in den Evidenztabellen nachlesbar (Supplement).
}

Serum $\geq 20 \mathrm{ng} / \mathrm{ml}$ ). Bedenken bezüglich potenziell negativer Auswirkungen von Kalzium- und Vitamin-D-Supplementierung auf das kardiovaskuläre Risiko beruhen auf Bobachtungsstudien und insbesondere einer Metaanalyse mehrerer RCTs, die ein um 31\% erhöhtes Risiko für kardiovaskuläre Ereignisse bei Kalziumsupplementation (ohne gleichzeitige Gabe von Vitamin D) ergab [16]. Obgleich eine RCT mit 36.282 postmenopausalen Frauen, die entweder Placebo oder 1000 mg Kalzium plus 400 IE Vitamin D täglich erhielten, keinen Effekt auf das kardiovaskuläre Risiko suggerierte [40], zeigte eine Subanalyse dieser Studie, dass ein erhöhtes Risiko nur bei Frauen bestand, die Kalziumsupplemente nach Randomisierung erhielten, und nicht bei Frauen, die bereits vor Randomisierung Kalzium einnahmen (gemäß Protokoll möglich) [17, 60]. Im Gegensatz zu den Bedenken, die im Zusammenhang mit Kalziumsupplementation geäußert wurden, zeigen prospektive Kohortenstudien entweder keinen [87, 93] oder einen günstigen Effekt $[46,55]$ bezüglich Kalziumzufuhr über die Nahrung auf das kardiovaskuläre Risiko, wobei keine RCTs zur finalen Bewertung des Risikos der Kalziumzufuhr mittels Supplementation vs. über Ernährung existieren. Es wird daher empfohlen, die Kalziumzufuhr primär über die Nahrung zu optimieren. Nur wenn die empfohlene Zufuhr von $1000 \mathrm{mg}$ Kalzium mit der Nahrung (z.B. kalziumhaltige Mineralwasser) nicht erreicht wird (ggf. Überprüfung der Kalziumzufuhr über Kalkulatoren/Fragebögen, z. B. einen im Internet verfügbaren Kalziumrechner, https://www.gesundheitsinformation. de/kalziumrechner/), sollte eine Supplementierung erfolgen.

Trotz Bedenken erscheint in der Abwägung von Nutzen und Risiko die Optimierung der Kalziumzufuhr bei GCbehandelten Patienten wichtig. Entscheidend ist dabei, dass GC insbesondere die renale Reabsorption von Kalzium reduzieren und die Kalziumausscheidung im Urin erhöhen, womit das im Serum verfügbare Kalzium verringert wird [21].
1. Was für einen/keinen Nutzen hat die Supplementation mit Kalzium bei GIOP?

- Die alleinige Supplementation von Kalzium ist zum Erhalt der BMD nicht ausreichend. (LoE 1b)

\section{Was für einen/keinen Nutzen hat die Supplementation mit Vitamin D bei GIOP?}

- Die alleinige Supplementation von Vitamin D ist vorteilhaft im Vergleich zur alleinigen Supplementation von Kalzium i.S. eines geringeren BMDVerlustes. (LoE 1b)

\section{Was für einen/keinen Nutzen hat die Supplementation mit Vitamin D und Kalzium bei GIOP?}

\section{- Die Supplementation von Vitamin D und Kalzium ist effektiv im Sinne eines geringeren BMD-Verlustes im Vergleich zu keiner Therapie oder Kalziumsupplementation. (LoE 1b)}

\section{Bisphosphonate}

Die Wirkmechanismen umfassen die Verringerung der osteoklastären Knochenresorption durch Anlagerung an Hydroxylapatitbindungsstellen auf Knochenoberflächen und insbesondere die Inhibition von Funktion, Entwicklung, Rekrutierung und Überleben von Osteoklasten durch Hemmung der für die Synthese von Farnesylpyrophosphat und Geranylgeranylpyrophosphat notwendigen Schlüsselenzyme des $\mathrm{Me}$ valonsäurezyklus. Zusätzlich scheinen Bisphosphonate die Inhibition der Apoptose bei Osteoblasten $\mathrm{zu}$ begünstigen, wobei dieser Mechanismus im Vergleich zur starken antiresorptiven Wirkung vermutlich nicht wesentlich zur antiosteoporotischen Wirksamkeit beiträgt, sodass es sich insgesamt um ein antiresorptives Medikament handelt.

Die Bisphosphonate erhöhen die BMD und senken das Risiko vertebraler und nichtvertebraler Frakturen bei GIOP-Patienten, wie in einer großen Metaanalyse zur Therapie der GIOP mit verschiedenen Bisphosphonaten ge- 


\begin{tabular}{|c|c|c|c|c|c|c|c|c|c|}
\hline & $\begin{array}{l}\text { Zulassung } \\
\text { GIOPa }\end{array}$ & Applikation GIOP & $\begin{array}{l}\text { Postmeno- } \\
\text { pausale } \\
\text { Osteopo- } \\
\text { rose Frau }\end{array}$ & $\begin{array}{l}\text { Osteo- } \\
\text { porose } \\
\text { Mann }\end{array}$ & $\begin{array}{l}\text { Anabol/ } \\
\text { anti- } \\
\text { resorptiv }\end{array}$ & $\begin{array}{l}\text { Zunahme BMD } \\
\text { LWS }\end{array}$ & $\begin{array}{l}\text { Zunahme } \\
\text { BMD } \\
\text { Femur }\end{array}$ & $\begin{array}{l}\text { Reduktion } \\
\text { Wirbel- } \\
\text { körper- } \\
\text { frakturen }\end{array}$ & $\begin{array}{l}\text { Reduktion } \\
\text { Femurfrak- } \\
\text { turen }\end{array}$ \\
\hline Kalzium/Vitamin D & + & & + & & NA & $\begin{array}{l}\text { Lediglich gerin- } \\
\text { gerer Rückgang }\end{array}$ & $\varnothing$ & $\varnothing$ & $\varnothing$ \\
\hline \multicolumn{10}{|l|}{ Bisphosphonate } \\
\hline Alendronat & + & 10 mg/Tag p.o. & + & + & $-/+$ & + & + & - & - \\
\hline Risedronat & $\begin{array}{l}+ \text { (nur } \\
\text { Frauen) }\end{array}$ & $5 \mathrm{mg} / \mathrm{Tag}$ & + & - & & + & + & + & $\varnothing$ \\
\hline Ibandronat & - & - & + & - & $-/+$ & + & + & $\varnothing$ & $\varnothing$ \\
\hline Pamidronat & - & - & + & $\begin{array}{l}+ \text { bei } \\
\text { (Tumor) }\end{array}$ & $-1+$ & $\varnothing$ & $\varnothing$ & $\varnothing$ & $\varnothing$ \\
\hline Zolendronat & + & $5 \mathrm{mg}$ 1-mal/Jahr i.v. & + & + & $-/+$ & + & + & $\varnothing$ & $\varnothing$ \\
\hline Teriparatid $^{\mathrm{b}}$ & + & $20 \mu \mathrm{g} / \mathrm{Tag}$ s.c. & + & + & $+/-$ & $++^{c}$ & - & + & $\begin{array}{l}\text { Kein sign. } \\
\text { Unterschied }\end{array}$ \\
\hline Denosumab & + & $\begin{array}{l}60 \mathrm{mg} \mathrm{1-mal} / 6 \mathrm{Mo}- \\
\text { nate }\end{array}$ & + & + & $-/+$ & ++ & ++ & $\varnothing$ & $\varnothing$ \\
\hline \multicolumn{10}{|c|}{$\begin{array}{l}\varnothing \text { Keine oder nicht suffiziente Daten verfügbar } \\
\text { GIOP Glukokortikoid-induzierte Osteoporose, BMD Knochenmineraldichte, i.v. intravenös, p.o. per os } \\
\text { aProphylaxe und Therapie der GIOP } \\
\text { 'Studie mit Teriparatid + Östrogen vs. Östrogen } \\
\text { 'Basierend auf Überlegenheit vs. Alendronat }\end{array}$} \\
\hline
\end{tabular}

zeigt wurde [8]. Diese Metaanalyse aus 27 RCTs ergab, dass Bisphosphonate sowohl ohne als auch in der Kombination mit Kalzium oder Vitamin D oder mit Kalzium plus Vitamin D das Risiko vertebraler und nichtvertebraler Frakturen senken und die BMD an der Wirbelsäule und am Femur im Vergleich zu Placebo erhöhen. Die Anwendung von Bisphosphonaten zur Therapie die GIOP ist damit grundsätzlich als sinnvoll zu erachten, wenngleich der Nutzen gemäß dieser Metaanalyse eher gering war. Der absolute Benefit in der Reduktion vertebraler Frakturen lag nach 12 bis 24 Monaten bei $2 \%$ (1-5\%; NNT 31) und in der Reduktion nichtvertebraler Frakturen bei $1 \%$. Im Rahmen der Interpretation der Daten muss zudem beachtet werden, dass als Einschlusskriterium nicht zwingend eine manifeste Osteoporose vorliegen musste, sondern lediglich das Vorliegen einer entzündlichen Erkrankung mit der Anwendung von GC (Dosis $\geq 5 \mathrm{mg} / \mathrm{Tag}$ ) und die Studiendauer (12 bis 24 Monate) sehr kurz waren. In diese Metaanalyse wurden auch Studien zu Bisphosphonaten aufgenommen, die keine Zulassung in Deutschland haben (z.B. Clodronat) [8]. Die Metaanalyse konnte keine wesentlichen Unterschiede bezüglich der
Nebenwirkungen in den Bisphosphonatvs. Placebogruppen feststellen [8].

\section{Einzelsubstanzen}

\section{Alendronat}

In 7 RCTs konnte für Frauen und Männer mit einer GIOP sowohl eine Zunahme der BMD an Lendenwirbelsäule und Femur festgestellt werden. Eine Reduktion des Auftretens von Wirbelkörperfrakturen durch den Einsatz von Alendronat konnte nicht einheitlich in den Studien erzielt werden [3, 62, 69, 82, 84].

Im Detail konnte für Alendronat $5 \mathrm{mg}$ und Alendronat $10 \mathrm{mg}$ vs. Placebo eine signifikante Zunahme der BMD an der Lendenwirbelsäule (Alendronat $5 \mathrm{mg}:+2,1 \%, p<0,001$; Alendronat $10 \mathrm{mg}$ : $+2,9 \%, p<0,001$; Placebo: $-0,4 \%$, $p=$ n.s.) und dem Femur (Alendronat $5 \mathrm{mg}:+1,2 \%, p<0,01$; Alendronat $10 \mathrm{mg}$ : $+1,0 \%, p<0,01$; Placebo: $-1,2 \%, p<0,01)$ über 48 Wochen nachgewiesen werden. Bezüglich des Auftretens neuer vertebraler und nichtvertebraler Frakturen lag kein signifikanter Unterschied zwischen den Alendronat-Gruppen und der Placebogruppe vor [69]. Ein ähnliches Ergebnis bezüglich der BMD wurde nach 2 Jahren im Rahmen der Extension-Stu- die evaluiert, wobei in der AlendronatGruppe (0,7\%) im Vergleich zur Placebo (Kalzium + Vitamin D)-Gruppe (6,8\%) signifikant $(p<0,05)$ weniger neue vertebrale Frakturen aufgetreten sind [3]. In einer kleinen Vergleichsstudie $(n=163)$ der Wirksamkeit einer Alendronat-Therapie vs. Placebo bezüglich der BMD und des Frakturrisikos wurde bei Patienten mit einer rheumatoiden Arthritis und einer dauerhaften GC-Therapie (Prednison: $<10 \mathrm{mg} / \mathrm{Tag}$ ) über 52 Wochen folgendes Ergebnis ermittelt: Zunahme der BMD an der Lendenwirbelsäule $(+3,7 \%)$ und am Femur $(+1,0 \%)$ für die Alendronat-Gruppe vs. einer Abnahme der BMD an der Lendenwirbelsäule $(-1,0 \%)$ und am Femur $(-0,1 \%)$. Hinsichtlich des Frakturrisikos traten in der Alendronat-Gruppe (13\%) mehr vertebrale Frakturen im Vergleich zu Placebo (4\%) auf, wobei kein signifikanter Gruppenunterschied bestand. Anzumerken ist, dass in der Studie die Patienten mit Alendronat in der Dosierung $5 \mathrm{mg}$ sowie $10 \mathrm{mg}$ je Tag therapiert wurden. In der Alendronat-10-mg-Dosierung vs. der Alendronat-5-mg-Dosierung traten 3 vs. 6 neue vertebrale Frakturen auf [53]. 
Alendronat in Kombination mit Vitamin D versus Alendronat versus Vitamin D. Ein RCT evaluierte bei Frauen $(n=106)$ mit einer entzündlich rheumatischen Erkrankung und einer GIOP die Wirksamkeit von Alendronat in Kombination mit Vitamin D im Vergleich zu Alendronat bzw. Vitamin D in der Monotherapie über 6 Monate. In der Kombinationsbehandlung von Alendronat und Vitamin D traten keine neuen Wirbelkörperfrakturen auf. In der Monotherapie mit Alendronat bzw. VitaminD-Gruppe traten 2 vs. 6 neue Wirbelkörperfrakturen im Untersuchungszeitraum auf [80]. Bezüglich der Details zu Applikation und Zulassungsstatus der einzelnen Bisphosphonate verweisen wir auf $\bullet$ Tab. 1.

\section{Risedronat}

Risedronat $5 \mathrm{mg}$ führte zu einer Stabilisierung bzw. einem Anstieg der BMD in 2 RCTs über je 12 Monate, in die sowohl Männer als auch Frauen eingeschlossen wurden, die eine GC-Therapie erhielten $[25,66]$. In einer Studie konnte zudem die Rate an Wirbelfrakturen durch Risedronat signifikant gegenüber Placebo um $70 \%$ reduziert werden [92]. ${ }^{2}$

\section{Ibandronat}

Es liegen 2 RCTs zur Behandlung der GIOP bei postmenopausalen Frauen vor, bei denen über 12 Monate bzw. 48 Monate eine Überlegenheit von Ibandronat (150 mg 1-mal monatlich per os) im Vergleich zu Placebo mit einer Zunahme der $\mathrm{BMD}$ an der Lendenwirbelsäule (Monat 12: Ibandronat $+3,2 \%$ vs. Placebo $-0,1 \%, p<0,001$; Monat 48 : Ibandronat $+4,8 \%$ vs. Placebo $-1,9 \% ; p<0,001)$ und am Femur (Monat 12: Ibandronat $+1,2 \%$ vs. Placebo $-0,7 \%, p<0,001$; Monat 48 : Ibandronat $+0,4 \%$ vs. Placebo $-1,9 \%$; $p<0,05)$ gezeigt werden konnte [38, 79]. Daten bezüglich einer Frakturreduktion sind nicht publiziert. ${ }^{2}$

\footnotetext{
2 Zulassungsstatus, Applikation s. - Tab. 1.
}

\section{Pamidronat}

Für Pamidronat liegen keine Daten für die Behandlung einer GIOP vor. ${ }^{3}$

\section{Zolendronat}

Während keine RCTs vorliegen, in denen Zolendronat gegenüber Placebo getestet wurde, existieren Vergleichsstudien mit anderen Bisphosphonaten bzw. anderen Substanzklassen (s. unten), die eine gute Wirksamkeit zeigten. ${ }^{4}$

\section{Was für einen/keinen Nutzen zeigt ein orales Bisphosphonat bei GIOP?}

- Alendronat, Ibandronat ${ }^{5}$ und Risedronat zeigen gegenüber Placebo eine Zunahme der BMD an der Lendenwirbelsäule und am Femur. (LoE 1b)

- Alendronat, Risedronat sind mit einer niedrigeren Frakturrate (Wirbelkörper) im Vergleich zu Placebo verbunden. (LoE 1b)

- Die alleinige Vitamin-D-Gabe ist im Vergleich zu Alendronat mit einem BMD-Verlust und einer höheren Rate an neuen Wirbelkörperfrakturen assoziiert. (LoE 2b)

- Die Kombinationstherapie mit Vitamin D und Alendronat zeigt im Vergleich zu den jeweiligen Monotherapien mit Vitamin D oder Alendronat die deutlichste Zunahme der BMD an der LWS und die niedrigste Frakturrate. (LoE 1b)

\section{Vergleichsstudien zwischen Bisphosphonaten}

In den letzten Jahren wurden mehrere vergleichende Studien der Bisphosphonate untereinander sowie zwischen Bisphosphonaten und anderen Substanzklassen publiziert.

Die meisten Studien mit Bisphosphonaten schlossen Patienten mit einer bereits bestehenden GC-Vortherapie

\footnotetext{
3 Zulassungsstatus, Applikation s. - Tab. 1.

4 Zulassungsstatus, Applikation s. DTab. 1.

${ }^{5}$ Cave keine GIOP-Zulassung.
}

(„Treatment Group“) oder einer beginnenden/geplanten GC-Therapie („Prevention Group") ein, ohne dass bereits eine Osteoporose (T-Score $\leq-2,5$ Standardabweichungen) vorlag, im Sinne eines präventiven Ansatzes.

\section{Zolendronat versus Risedronat}

Zolendronat war in 2 RCTs über 12 Monate der Anwendung von Risedronat ( $5 \mathrm{mg} / \mathrm{Tag}$ ) bezüglich der Zunahme der BMD der LWS (Zolendronat: 4,7 und $4,1 \%$ vs. Risedronat: $3,3 \%$ vs. $2,7 \%$; $p<0,05)$ überlegen [65, 77]. Kritisch anzumerken ist hier, dass Patienten mit einer nur gering reduzierten Knochendichte (T-Score minimal bis -1,48 Standardabweichungen) eingeschlossen wurden. Zudem gab es keinen signifikanten Unterschied in der Rate vertebraler Frakturen (kombinierte Analyse beider Subgruppen „Prevention“- und „Treatment“Gruppe) [61] bei allerdings kurzem Beobachtungszeitraum.

Weitere Vergleichsstudien zu anderen Bisphosphonaten liegen nicht vor.

\section{Was für einen/keinen Nutzen zeigt ein intravenöses Bisphosphonat bei GIOP}

- Der Zusatznutzen einer intravenösen Bisphosphonat-Therapie bei einer GIOP kann anhand der vorhandenen Daten nicht klar belegt werden, wobei die deutlichere Zunahme der BMD für einen Einsatz von Zolendronat gegenüber Risedronat spricht. (LoE 1b)

\section{Ist eine orale Bisphosphonat- Therapie einer intravenösen Bisphosphonat-Therapie bei GIOP überlegen?}

- Die Fragestellung kann anhand der Datenlage nicht abschließend beantwortet werden. Es gibt eine gewisse Tendenz, dass Zolendronat im Vergleich zu Risedronat zu einer signifikant höheren Zunahme der BMD führt. Studien mit dem Frakturrisiko als primärer Endpunkt sind nicht publiziert. Weitere Vergleichsstudien liegen ebenfalls nicht vor. (LoE 1b) 
Vergleichsstudien zwischen

Bisphosphonaten und anderen Substanzklassen

\section{Risedronat versus Vitamin D}

Risedronat war in einem sehr kleinen RCT $(n=12)$ in einer Dosis von $2,5 \mathrm{mg} / \mathrm{Tag}$ in Kombination mit Kalzium $800 \mathrm{mg} /$ Tag über 12 Monate einer Therapie mit Alfacalcidol 0,5 $\mu$ g/Tag in Kombination mit Kalzium $800 \mathrm{mg} / \mathrm{Tag}$ hinsichtlich der Zunahme der BMD überlegen. Diese Studie ist klein und untersuchte keine harten Endpunkte (z.B. Reduktion des Frakturrisikos), ist aber erwähnenswert, da hier ausschließlich RA-Patienten mit einem T-Score $\leq-2,5$ Standardabweichungen eingeschlossen wurden, d. h. Patienten mit manifester GIOP [94].

\section{Risedronat versus Denosumab}

Risedronat war Denosumab in einigen Endpunkten unterlegen (s. unten) [71, 73].

\section{Alendronat versus Teriparatid}

In der Behandlung der GIOP zeigte sich Teriparatid im Vergleich zu Alendronat überlegen (s. unten) [72, 74].

\section{Teriparatid}

Teriparatid ist das aktive, mit biotechnologischen Methoden hergestellte Fragment des endogenen humanen Parathormons, das aus den ersten 34 Aminosäuren des körpereigenen Parathormons besteht. Obwohl eine dauerhafte Erhöhung des Parathormonspiegels (durch Aktivierung von RANKL und Abfall von Osteoprotegerin) eine persistierende Osteoklastenstimulation zur Folge hat, kann durch tägliche, subkutane Injektion von rekombinantem PTH die Bildung neuen Knochens durch Steigerung des Knochenremodellings erhöht sowie eine $\mathrm{Zu}$ nahme der trabekulären Vernetzung und der Dicke von kortikalem Knochen induziert werden.

Im Unterschied zu Bisphosphonaten und Denosumab hemmt es nicht den Knochenabbau, sondern fördert den Aufbau (anabole Wirkung) durch di- rekte Stimulation der Osteoblasten. Es erhöht zudem die Absorption von Kalzium im Gastrointestinaltrakt und fördert dessen renale Reabsorption sowie die renale Phosphatausscheidung. ${ }^{6}$

\section{Studien zur Wirkung bei GIOP}

\section{a) Kontrollierter Vergleich mit Alendronat}

Bereits 1998 wurde ein RCT mit Vergleich Teriparatid + Östrogen vs. Östrogen publiziert, in dem sich signifikante Vorteile für die Teriparatid-Gruppe in Bezug auf die Knochendichte an der LWS (aber nicht an Femur und Unterarm) zeigten [49]. Später folgten dann die Ergebnisse eines über 18 Monate laufenden randomisiert kontrollierten Vergleiches zwischen täglicher Gabe von $20 \mu \mathrm{g}$ Teriparatid und $10 \mathrm{mg}$ Alendronat [72]. Bei Untersuchung von 428 Patienten/ Patientinnen mit Osteoporose und $\geq 5 \mathrm{mg}$ Prednisolon seit $\geq 3$ Monaten erwies sich Teriparatid bezüglich des primären Endpunkts, Veränderung der BMD an der LWS als signifikant überlegen $(7,2 \%$ vs. $3,4 \%, p<0,001)$, wobei sich der signifikante Unterschied bereits nach 6 Monaten zeigte. Auch am Femur nahm die BMD unter Teriparatid signifikant mehr zu $(3,8 \%$ vs. $2,4 \%, p=0,005)$. Vertebrale Frakturen traten ebenfalls signifikant seltener auf ( 1 vs. 10, $p=0,004)$, während sich die Inzidenz nonvertebraler Frakturen nicht unterschied (12 vs. $8, p=0,36$ ).

In einer weiteren Publikation wurde über die Extension dieses RCT auf 36 Monate berichtet [74]. Der signifikante Vorteil von Teriparatid zeigte sich auch im Gesamtzeitraum, so lag der BMD-Zuwachs nach 36 Monaten an der LWS bei $11 \%$ vs. $5,3 \%$, an Hüfte bei $5,2 \%$ vs. $2,7 \%$ und am Femur bei $6,3 \%$ vs. $3,4 \%$ (jeweils $p<0,001$ ). Vertebrale Frakturen traten in 3 vs. 13 Fällen auf (1,7\% vs. $7,7 \%, p=0,007)$. Auch in diesem Zeitraum war die Häufigkeit nonvertebraler Frakturen in beiden Gruppen gleich. In einer Ergänzungsuntersuchung wurde der trabekuläre Bone Score ermittelt, der nur unter Teriparatid signifikant um $3,7 \%(p<0,05)$ zunahm [68].

\footnotetext{
${ }^{6}$ Zulassungsstatus, Applikation s. - Tab. 1.
}

\section{b) Weitere Vergleichsstudien mit Bisphosphonaten}

In einer über 18 Monate laufenden offenen randomisierten Studie wurden 92 Männer unter GC-Therapie seit $\geq 3$ Monaten (mediane Dosis $8,8 \mathrm{mg} / \mathrm{Tag}$ ) und mit $\mathrm{T}$-Score $\leq 1,5 \mathrm{SD}$ mit $20 \mu \mathrm{g}$ Teriparatid vs. $35 \mathrm{mg} /$ Woche Risedronat behandelt [35]. Die Zunahme der Knochendichte lag unter Teriparatid bei $16,3 \%$, unter Risedronat bei 3,8\% $(p=0,004)$. Neue klinisch manifeste Frakturen traten unter Teriparatid in keinem, unter Risedronat in 5 Fällen auf $(p=0,056)$.

\section{c) Weitere Studien}

In einer offenen Beobachtungsstudie wurden 1581 postmenopausale Patientinnen mit Osteoporose für 18 Monate mit Teriparatid und dann über weitere 18 Monate mit anderen Osteoporosemedikationen behandelt [45]. Eine Subgruppe von 294 Patientinnen stand unter GC-Dauertherapie, 69 Frakturen traten bei diesen Patientinnen im Gesamtverlauf auf. Im Vergleich $\mathrm{zu}$ den ersten 6 Monaten unter Teriparatid nahm die Frakturrate im Zeitraum 24 bis $30 \mathrm{Mo}-$ nate um $81 \%$, 30 bis 36 Monate um $89 \%$ $\mathrm{ab}(p$ jeweils $<0,05)$. Die Frakturinzidenz sank von 1272/10.000 Patientenjahre im ersten bis auf 120/10.000 Patientenjahre im letzten Halbjahr. Parallel nahmen Rückenschmerzen signifikant $a b$, die Lebensqualität (gemessen mit Healthrelated quality of life-Fragebogen) signifikant zu.

Eine integrative Analyse aus 4 prospektiven Beobachtungsstudien enthielt eine Subgruppenauswertung von $958 \mathrm{~Pa}$ tienten mit GC-induzierter Osteoporose [50]. Wie in den anderen Subgruppen zeigte sich auch bei den GC-Patienten eine stabile und signifikante Reduktion der Rate vertebraler Frakturen ab Monat 6 und bis Monat 24 im Vergleich zum ersten Halbjahr der Teriparatid-Therapie, hingegen nicht bei den nonvertebralen Frakturen.

\section{Was für einen/keinen Nutzen zeigt Teriparatid bei GIOP?}

- Teriparatid ist bei GIOP als stärker wirksam als Alendronat in Bezug 
auf Zunahme der BMD an LWS und Femur und auf die Frakturrate an der LWS (aber nicht nonvertebraler Frakturen) einzuschätzen. (LoE $1 \mathrm{~b}$ )

8. Ist eine osteoanabole Therapie mit Teriparatid einer Bisphosphonat-Therapie bei GIOP überlegen?

- Die Therapie mit Teriparatid zeigt im Vergleich zu Alendronat eine signifikantere und deutlichere Zunahme der BMD an der LWS und am Femur. (LoE 1b)

- Teriparatid zeigt eine niedrigere Rate neuer Wirbelkörperfrakturen im Vergleich zu Alendronat. (LoE 1b)

- Bezüglich nichtvertebraler Frakturen besteht zwischen Teriparatid und Alendronat kein signifikanter Unterschied. (LoE 1b)

\section{Ist eine osteoanabole Therapie} mit Teriparatid einer DenosumabTherapie bei GIOP überlegen?

- Es liegt bisher keine Studie mit einem Vergleich zwischen Teriparatid und Denosumab bei GIOP vor.

\section{Denosumab}

Denosumab ist ein humaner monoklonaler Antikörper, der hochaffin an den Receptor Activator of NF- $\kappa B$ Ligand (RANKL) bindet und damit die Bildung, Aktivierung und das Überleben von Osteoklasten inhibiert und damit $\mathrm{zu}$ einer reduzierten Resorption von trabekulären und kortikalen Knochen führt. Es handelt sich somit um eine antiresorptive Substanz. ${ }^{\text {? }}$

\section{Studien zur Wirkung bei GIOP}

\section{a) Placebokontrolle}

In einer randomisiert doppelblinden placebokontrollierten Phase-2-Studie erhielt eine Subgruppe von $90 \mathrm{RA}-\mathrm{Pa}$ tienten unter initial im Median $5 \mathrm{mg}$ Prednisolon entweder Placebo oder Denosumab in Zulassungsdosierung von

\footnotetext{
7 Zulassungsstatus, Applikation s. - Tab. 1.
}

$60 \mathrm{mg}$ zu Beginn und nach 6 Monaten. Nicht eingegangen wird an dieser Stelle auf eine weitere Gruppe, die die nicht zugelassene Dosierung von $180 \mathrm{mg}$ erhielt, sowie auf eine weitere Gruppe mit begleitender Bisphosphonat-Gabe [30]. Patienten unter $60 \mathrm{mg}$ Denosumab boten im Vergleich zu Placebo einen statistisch signifikanten BMD-Anstieg sowohl in der LWS als auch am Femur. Numerisch stieg die BMD um 3,5 $\pm 2,9 \%$ vs. $0,4 \pm 3,7 \%$ bzw. um $1,6 \pm 1,9 \%$ vs. $-1,2 \pm 2,6 \%$, Frakturen wurden in dieser Studie nicht erfasst.

\section{b) Kontrollierter Vergleich mit Risedronat}

In einer randomisiert doppelblinden kontrollierten Studie erhielten 795 Patienten unter einer Therapie mit $\geq 7,5 \mathrm{mg}$ Prednisolon (mittlere Dosis $14 \mathrm{mg}$ ) über 24 Monate halbjährlich $60 \mathrm{mg}$ Denosumab oder $5 \mathrm{mg}$ Risedronat täglich. Einschlusskriterium war für Patienten unter 50 Jahren die Anamnese einer GCinduzierten Fraktur, über 50 ein T-Score von mindestens $-2,0$ oder $-1,0+$ eine Fraktur [71, 73]. Es standen 505 Patienten unter GC-Dauertherapie (Gr. A), 290 begannen diese Therapie (Gr. B); $77 \%$ wiesen eine entzündlich rheumatische Erkrankung auf; 74,2\% der Patienten wurden über den gesamten Zeitraum von 2 Jahren behandelt.

Alle BMD-Unterschiede waren signifikant zugunsten von Denosumab: An der LWS in Gr. A $6,4 \%$ vs. 3,2\%, in Gr. B 6,2\% vs. 1,7\%, am Femur 2,9\% vs. $0,5 \%$ bzw. $3,1 \%$ vs. $0,0 \%$. Eine Auswertung der Frakturrate war vom Design her nicht vorgesehen, unter Denosumab traten 26, unter Risedronat 23 Frakturen auf, der Unterschied war nicht signifikant $[71,73]$.

\section{c) Weitere Vergleichsstudien mit Bisphosphonaten}

In einem 1-jährigen offenen RCT wurde bei 42 an RA oder SLE leidenden Patientinnen unter Langzeit-GC-Therapie $(4,4 \mathrm{mg})$ und unter Bisphosphonat (79\% Alendronat, 12\% Risedronat, $10 \%$ Ibandronat) entweder die Bisphosphonat-Therapie fortgesetzt oder auf Denosumab gewechselt [61]. Nach 1 Jahr lag die BMD an der Wirbelsäule bei $+1,5 \%$
( $p=0,001)$ vs. $+3,4 \%(p=0,002)$, am Femur bei $+0,80 \%(p=0,12)$ vs. $+1,4 \%$ $(p=0,03)$. Auch in dieser Studie war die Frakturrate nicht Gegenstand der Auswertungen, es traten in beiden Gruppen auch keine Frakturen auf.

In einer weiteren 1-jährigen prospektiven, offenen randomisiert kontrollierten Studie wurde bei 32 Patienten mit glomerulärer Erkrankung und GIOP die Therapie mit Denosumab bzw. mit Alendronat verglichen [42]. Hier war nur der BMD-Unterschied an der LWS signifikant zugunsten von Denosumab (+5,3\% vs. $+2,0 \%, p<0,05)$. Am Femur und am Radius zeigten sich hingegen keine signifikanten Unterschiede. In der Denosumab-Gruppe trat eine, unter Alendronat keine Fraktur auf.

\section{d) Systematischer Review mit Metaanalyse}

Ein systematischer Review mit Metaanalyse der genannten Studien ermittelte eine Überlegenheit von Denosumab im Vergleich zu Bisphosphonaten bezüglich der Knochendichte an der LWS und am Femur [95]. Alle Studien wiesen keine Power zur Erfassung von Unterschieden in der Frakturrate auf.

\section{Was für einen/keinen Nutzen zeigt Denosumab bei GIOP}

- Denosumab führt bei GIOP zu einer signifikanten Zunahme der BMD an LWS und Femur im Vergleich zu Placebo. Der Effekt auf die Frakturrate wurde nicht untersucht. (LoE 1b)

\section{Ist eine Therapie mit}

Denosumab einer BisphosphonatTherapie bei GIOP überlegen?

- Denosumab ist bei GIOP einer Bisphosphonat-Therapie bezüglich der BMD an LWS und Femur überlegen. Ein Vorteil bezüglich Frakturrate wurde bisher nicht belegt. (LoE 1b) 


\section{Welche antiresorptiven und osteoanabolen Therapieverfahren können bei einer chronischen Niereninsuffizienz bei GIOP eingesetzt werden?}

- Entsprechend der Datenlage und dem Zulassungsstatus kann bei einer chronischen Niereninsuffizienz (GFR $<30 \mathrm{ml} / \mathrm{min}$ ) zur Behandlung einer GIOP nur Denosumab eingesetzt werden, wobei eine renale Osteopathie ausgeschlossen werden sollte. (Siehe Fachinformationen der einzelnen Substanzen)

\section{Sicherheitsaspekte und Therapie in besonderen Situationen}

\section{Kalzium und Vitamin D}

Vitamin D sollte mit Vorsicht bei Patienten mit einer chronischen Niereninsuffizienz, Sarkoidose, Immobilisation, Neigung zu kalziumhaltigen Nierensteinen und bei Patienten mit gestörter renaler Kalzium- und Phosphatausscheidung, Pseudohypoparathyreoidismus und bei Behandlung mit Benzothiadiazin-Derivaten angewendet werden. Der Kalziumspiegel sollte im Plasma und Urin überwacht werden. Bei chronischer Niereninsuffizienz können andere Empfehlungen bezüglich Vitamin-D-Gabe bestehen, weswegen wir diesbezüglich auf die nephrologischen Leitlinien verweisen (KDIGO) [47].

\section{Bisphosphonate}

\section{Alter}

Der Einsatz von Bisphosphonaten wird bei Kindern und Jugendlichen unter 18 Jahren aufgrund einer unzureichenden Datenlage hinsichtlich Effektivität und Nebenwirkungen nicht empfohlen. Die Datenlage zur Anwendung von Bisphosphonaten bei hochbetagten Frauen (>80 Jahre) ist begrenzt. Möglicherweise besteht im höheren Lebensalter ein geringerer Schutz vor Frakturen durch die Anwendung von Risedronat. ${ }^{8}$

${ }^{8}$ Fachinformationen Risedronat, Stand 06/2018, Zolendronat, Stand 07/2019.

\section{Schwangerschaft und Stillzeit}

Bisphosphonate sind in der Schwangerschaft und Stillzeit kontraindiziert.

\section{Chronische Niereninsuffizienz}

Bei Patienten mit einer GIOP und einer ausgeprägten chronischen Niereninsuffizienz ist der Einsatz von Bisphosphonaten kontraindiziert (GFR $<30 \mathrm{ml} / \mathrm{min}$ : Pamidronat, Risedronat; GFR $<35 \mathrm{ml} / \mathrm{min}$ : Alendronat, Zoledronat), oder es muss eine Dosisreduktion erfolgen (Ibandronat). In dieser Situation steht als einzig zugelassenes Therapieverfahren Denosumab zur Verfügung.

\section{Hypokalziämie und Hypovitami-} nose D sowie Funktionsstörungen der Nebenschilddrüse

Eine Hypokalziämie stellt eine Kontraindikation für die Anwendung von Bisphosphonaten dar. Hypokalziämien, Vitamin-D-Mangelzustände und Störungen der Nebenschilddrüsenfunktion sind vor Beginn einer Bisphosphonat-Therapie zu diagnostizieren und zu behandeln.

\section{Osteonekrosen des Kiefers}

Kiefernekrosen sind eine seltene Komplikation unter Bisphosphonaten bei GIOP und treten häufiger bei Langzeit-Bisphosphonattherapie auf [34]. Ihre Häufigkeit wird mit $1 \mathrm{zu} 100.000$ angegeben [1]. Vor Beginn einer Therapie mit Bisphosphonaten sollen bei Patienten mit begleitenden Risikofaktoren daher eine zahnärztliche Untersuchung und ggf. eine zahnärztliche Präventivbehandlung durchgeführt werden. Unter dieser Therapie sollten zahnärztliche Kontrolluntersuchungen regelmäßig wiederholt werden [1, $14]$.

\section{Atypische Femurfrakturen}

Unter Bisphosphonaten wurden - insbesondere bei Langzeitbehandlungen atypische subtrochantäre und diaphysäre Femurfrakturen beobachtet. Die Inzidenz wird mit 6 bis 30/10.000 Patientenjahren beziffert [1]. Atypische Femurfrakturen können offensichtlich oh- ne oder auch nach minimalen Traumata entstehen und treten häufig bilateral auf. ${ }^{9}$

In den jeweiligen Fachinformationen und bestimmten Empfehlungen wird dazu geraten, ein Absetzen der Bisphosphonat-Therapie nach einer atypischen Femurfraktur anhand einer individuellen Nutzen-Risiko-Bewertung zu erwägen (s. unten).

Aufgrund des möglichen bilateralen Auftretens dieser Frakturen sollte immer auch das kontralaterale Femur untersucht werden. In diesen Situationen sollte eine osteoanabole Therapie bedacht werden, wobei aktuell keine sichere Evidenz für diese Therapiesituation vorliegt.

Hinsichtlich der Nebenwirkungen wurde in einer Studie eine höhere Rate an grippeähnlichen Symptomen und Pyrexie im Zolendronat-Arm detektiert [73].

\section{Therapiedauer von Bisphosphonaten}

Aufgrund des erhöhten Risikos von atypischen Femurfrakturen und Kiefernekrosen unter Langzeit-Bisphosphonattherapie rät die amerikanische „Endokrine Society“ in Bezug auf postmenopausale Frauen unter einer Bisphosphonat-Therapie, das Frakturrisiko nach 3 bis 5 Jahren Therapie zu reevaluieren und bei Patientinnen mit einem weiterhin hohen Frakturrisiko die Therapie fortzusetzen. Bei Patientinnen mit einem niedrigen bis moderaten Frakturrisiko kann ein Absetzen der Medikation („drug holiday“) für 3 bis 5 Jahre in Betracht gezogen werden [31]. Auch die deutsche DVO-Leitlinie (2017) empfiehlt eine Reevaluation der spezifischen osteologischen Therapie nach einer Therapiedauer von 3 bis 5 Jahren hinsichtlich des Risikos sowie des Nutzens und gibt den Hinweis, dass eine generelle Begrenzung der Therapie mit Bisphosphonaten auf 3 bis 5 Jahre nicht besteht. Weitere spezifische Empfehlungen werden in der DVO-Leitlinie nicht gegeben [1].

In einer systematischen Übersichtsarbeit, welche die Vor- und Nachteile einer

${ }^{9}$ Fachinformationen Teriparatid, Stand $11 / 2017$. 
Langzeittherapie mit Bisphosphonaten (3 bis 5 Jahre) und der weiteren Fortführung über diesen Zeitraum hinaus gegenüber dem Absetzen von Bisphosphonaten („drug holiday“) analysiert, stellten sich eine Reduktion von radiologischen Wirbelkörperfrakturen bei der Fortführung von Zolendronat sowie eine Reduktion klinischer Wirbelkörperfrakturen bei der Fortführung von Alendronat dar. Eine Reduktion nichtvertebraler Frakturen wurde nicht eruiert. Grundsätzlich wird eine erhöhte Rate von atypischen Femurfrakturen und Kiefernekrosen bei der Langzeitanwendung von Bisphosphonaten bestätigt. Bei einer Fortführung von Alendronat oder Zolendronat über den Zeitraum von 3 bis 5 Jahren hinaus (vs. Absetzen) zeigten sich keine signifikanten Unterschiede bezüglich schwerer Nebenwirkungen, wobei die entsprechenden prospektiven RCTs eine zu geringe Anzahl an atypischen Femurfrakturen und Kiefernekrosen aufwiesen, um daraus Schlussfolgerungen zu ziehen. Eine retrospektive Studie wies eine erhöhte Rate von atypischen Femurfrakturen bei fortgeführter Bisphosphonat-Therapie (vs. Absetzen) auf (0,15\% vs. 0,03\%, OR: 6,03 [KI 1,87-19,42]). Die absolute Steigerung der Frakturrate betrug $0,13 \%$ [KI: 0,08-0,19\%], wobei potenzielle Confounder nicht berücksichtigt wurden [6].

Auf der Basis der ausgewerteten Studien des Reviews folgern die Autoren hinsichtlich einer Langzeittherapie mit Bisphosphonaten, dass pro 1000 postmenopausalen Frauen mit Osteoporose, die entweder mit Alendronat für 4 Jahre (vs. Placebo) oder mit Zolendronat für 6 Jahre (vs. Placebo) behandelt werden, bei 50 bis 70 zusätzlichen Frauen eine klinisch evidente Fraktur verhindert wird und dabei 2 zusätzliche Frauen eine subtrochantäre bzw. Femurfraktur erleiden. Pro 1000 Frauen, die bereits eine Vorbehandlung mit Bisphosphonaten aufweisen, wird berechnet, dass bei einer 3- bis 5-jährigen Vortherapie mit Alendronat eine Fortführung dieser Therapie für weitere 3 bis 5 Jahre (vs. Absetzen der Therapie) keine zusätzliche nichtvertebrale Faktur, aber 30 zusätzliche Wirbelkörperfrakturen verhindert werden; dabei wür- de eine zusätzliche subtrochantäre bzw. Femurfraktur auftreten.

Die Autoren weisen darauf hin, dass die Stärke der Evidenz für diese Feststellungen gering ist sowie keine Daten für Männer vorliegen und die Methoden zur Feststellung von Nebenwirkungen sehr heterogen waren [34]. Ebenso ist zu beachten, dass diese Studien nicht speziell auf die Untersuchung von GIOP-Patienten ausgelegt waren. Eine Langzeit-GCTherapie könnte einen wesentlichen Einfluss auf die generelle Erhöhung der Frakturrate haben; es ist daher denkbar, dass Bisphosphonate bei GIOP einen höheren Nutzen in der Protektion vor Frakturen haben.

Insgesamt kann aus der bisher vorliegenden Datenlage in Bezug auf die postmenopausale Osteoporose gefolgert werden, dass sowohl für eine Langzeitanwendung von Bisphosphonaten über einen Zeitraum von 3 bis 5 Jahren als auch für eine weitere Fortführung über diesen Zeitraum hinaus ein eher geringer zusätzlicher Nutzen besteht. Allerdings scheint das Risiko für eine Langzeittherapie hinsichtlich des Auftretens atypischer Femurfrakturen demgegenüber noch deutlich geringer zu sein. Zusammengefasst scheint die Anwendung der Bisphosphonate bis 5 Jahre sicher zu sein, wobei bei hohem Frakturrisiko eine längere Therapiedauer auf der Basis einer individuellen Entscheidung erwogen werden kann.

\section{Zustand nach Organ- transplantation}

Die Anwendung von Risedronat oder Zoledronat gegenüber Placebo war mehrheitlich mit einer Stabilisierung oder einem Anstieg der BMD bei Patienten nach Herz-, Lungen- oder Lebertransplantation nach 12 Monaten assoziiert, die eine GFR $>30 \mathrm{ml} / \mathrm{min}$ aufwiesen und sich unter einer immunsuppressiven Therapie (unter anderem mit GC) befanden [24, 27, 37, 78, 85]. In einer Vergleichsstudie von Alendronat und Zoledronat ergaben sich geringe Vorteile für Zoledronat bezüglich des Anstiegs der BMD in der Wirbelsäule [78]. Die meisten Patienten wiesen zum Zeitpunkt des Studienbeginns keine manifeste Osteoporo- se auf, sondern hatten eine normale bzw. osteopene Knochenmineraldichte.

Sofern keine hochgradige Einschränkung der Nierenfunktion besteht, können diese Bisphosphonate bei organtransplantierten Patienten für 12 Monate angewendet werden.

\section{Neue osteoporotische Majorfraktur unter einer antiresorptiven Therapie}

Daten zur Therapieänderung des Therapieregimes bei einer neu aufgetretenen osteoporotischen Majorfraktur (klinische Wirbelkörperfraktur, Hüftfraktur, Oberarmfrakturen, Unterarmfrakturen) unter einer antiresorptiven Therapie liegen nicht vor.

Als Risikofaktoren für das Auftreten von Majorfrakturen gelten die kumulative GC-Dosis, die Dauer der GC-Einnahme sowie die rheumatoide Arthritis und Polymyalgia rheumatica, welche besonders durch eine Erniedrigung der BMD gekennzeichnet ist.

\section{Anmerkung}

Anhand der vorliegenden Studien ist eine Differenzierung der therapeutischen Optionen zwischen Mann und Frau nicht sicher möglich. In den alten Studien wird häufig als Endpunkt die BMD herangezogen. Der harte Endpunkt mit der Reduktion von neuen vertebralen und nichtvertebralen Frakturen wird nur in den neueren Studien genutzt.

\section{Teriparatid}

Eine ausreichende Versorgung mit Kalzium und Vitamin D ist unter der Therapie mit TER wichtig. Eine vorbestehende Hyperkalzämie vor Therapiebeginn stellt eine Kontraindikation dar. Ebenso sollte die Substanz bei schwerer Niereninsuffizienz nicht, bei mittelschwerer mit Vorsicht angewendet werden, eine leichte Niereninsuffizienz stellt keine Einschränkung dar. In der Schwangerschaft und Stillzeit ist TER kontraindiziert. Auch im Kindes- und Jugendalter sollte es nicht angewendet werden. Eine Dosisanpassung in höherem Lebensalter ist nicht notwendig. Metabolische 
Knochenerkrankungen wie Hyperparathyreoidismus oder Morbus Paget und Malignome des Skelettes (einschließlich ossäre Metastasen) stellen eine Kontraindikation für die Anwendung dar ${ }^{10}$.

$\mathrm{Da}$ es bei Studien in Ratten bei Langzeitanwendung von TER zu einer erhöhten Inzidenz von Osteosarkomen kam, sollte nach gegenwärtigem Stand des Wissens die Behandlungsdauer mit dieser Substanz maximal 2 Jahre betragen. Allerdings ergab die Zwischenauswertung einer auf 15 Jahre angelegten Langzeitbeobachtungsstudie mit dem Ziel, das Osteosarkomrisiko beim Menschen zu ermitteln, nach 7 Jahren keinen Hinweis darauf, dass ein solches Risiko tatsächlich besteht [11]. Ansonsten bot TER in der großen Vergleichsstudie mit Alendronat [74] eine ähnlich gute Verträglichkeit, in beiden Gruppen kam es kaum zu schweren unerwünschten Ereignissen.

\section{Denosumab}

Eine ausreichende Versorgung mit Kalzium und Vitamin D ist unter der Therapie mit Denosumab wichtig und eine Hypokalzämie vor Therapiebeginn auszuschließen. Vor jeder Anwendung sollte der Kalziumspiegel überprüft werden. Eine gleichzeitige GC-Therapie stellt ein zusätzliches Risiko für Hypokalzämie dar ${ }^{11}$.

Weder bei Patienten >65 Jahre noch bei eingeschränkter Nierenfunktion ist bei DEN eine Dosisanpassung notwendig. Bei einer GFR $<30 \mathrm{ml} / \mathrm{min}$ ist besonders auf das erhöhte Risiko einer Hypokalzämie zu achten. In der Schwangerschaft ist die Denosumab-Anwendung nicht empfohlen. Bezüglich der Stillzeit ist eine sorgfältige Nutzen-Risiko-Abwägung vorzunehmen. Im Kindes- und Jugendalter sollte Denosumab nicht angewendet werden.

10 Fachinformationen Teriparatid, Stand

$11 / 2017$.

11 Fachinformationen Prolia ${ }^{\circledR}$ (Amgen $\mathrm{GmbH}$, Amgen Europe B.V., Breda, Niederlande), Stand 01/2020.

\section{Verträglichkeit}

Im Gegensatz zu den Studien bei postmenopausalen Frauen bot Denosumab in den Studien bei GIOP keine Hinweise für ein erhöhtes Infektionsrisiko, wie die Auswertung in der oben genannten Metaanalyse zeigte, die Risk Ratio lag hier bei 2,16 [95].

Besondere Aufmerksamkeit erfordert die Anwendung von Denosumab wegen des Risikos einer stark erhöhten Rate an Knochenresorption mit erhöhter Frakturrate nach Absetzen [28]. Eine Post-hoc-Analyse aus der bei postmenopausalen Patientinnen durchgeführten FREEDOM-Studie zeigte, dass nach Absetzen von Denosumab die Frakturrate von 1,2/100 Patientenjahre auf 7,1 anstieg. Entsprechende Untersuchungen liegen für die GIOP bisher nicht vor. Unter Berücksichtigung der Daten der FREEDOM-Studie sollte nach Beendigung der Therapie mit Denosumab auch bei der GIOP ein weiteres antiresorptives Therapieverfahren (z. B. Bisphosphonat) angeschlossen werden. Das Auftreten einer Kieferosteonekrose unter Denosumab wurde in Studien sehr selten beobachtet (16 Fälle bei 3148 Patienten, s. Fachinfo). Wie hoch das Risiko bei Behandlung der GIOP ist, wurde bisher nicht separat untersucht. Gegebenenfalls sollte in Abhängigkeit von etwaigen klinischen Konstellationen eine zahnärztliche Untersuchung vor Einleitung einer Therapie mit Denosumab durchgeführt werden.

\section{Romosozumab}

Neu in der Therapie ist Romosozumab als Antikörper gegen Sclerostin in der Behandlung der Osteoporose zugelassen. Daten bzw. klinische Studien zur Behandlung der GIOP liegen noch nicht vor, sodass aktuell keine Empfehlung zum Einsatz von Romosozumab in der Indikation der GIOP gegeben werden kann.

\section{Empfehlungen GIOP}

\section{Übergeordnete Prinzipien}

1. Bei allen Patienten unter GC-Langzeittherapie (>3 Monate) plus
1 Risikofaktor ${ }^{12}$ soll ein Risikoassessment inklusive BMD-Messung erfolgen.

2. Bei Erwachsenen unter geplanter GCLangzeittherapie sollte eine tägliche Zufuhr von $1000 \mathrm{mg} / \mathrm{Tag}$ Kalzium und $800 \mathrm{IE} /$ Tag Vitamin D erfolgen.

3. Bei Erwachsenen mit erhöhtem Frakturrisiko gemäß DVO-Definition sollte zusätzlich zu Kalzium und Vitamin D eine spezifische osteologische Therapie zur Anwendung kommen.

\section{Empfehlungen}

1. Wenn die Indikation für den Einsatz einer spezifischen osteologischen Therapie besteht, empfehlen wir Bisphosphonate oder Denosumab als Erstlinientherapie.

2. Innerhalb der Gruppe der zugelassenen Bisphosphonate kann keine eindeutige Präferenz bezüglich einer Substanz oder Applikationsform empfohlen werden, da für die verschiedenen Bisphosphonate ein unterschiedlicher Zulassungsstatus vorliegt und dieser im Rahmen der Verordnung zu beachten ist.

3. Wir empfehlen Teriparatid als primäre spezifische osteologische Therapie bei hohem Frakturrisiko, wobei die zugelassene Therapiezeit 2 Jahre beträgt.

4. Für Patienten, die das Therapieziel nicht erreicht haben ${ }^{13}$, sollte die Umstellung auf eine andere Klasse spezifischer osteologischer Medikamente erfolgen (Anmerkung: bei Compliance-Problemen oder eingeschränkter gastrointestinaler Resorption ist eine Umstellung der oralen Applikationsform z. B. auf eine intravenöse Gabe zu favorisieren).

5. Wir empfehlen eine Reevaluation bezüglich Risiken nach einer Therapiedauer von 3 bis 5 Jahren oder nach Beendigung der Langzeit-GCTherapie.

\footnotetext{
12 GemäßDVO-Leitlinie (2017).

13 z. B. neue Fraktur $\geq 18$ Monate nach Beginn der Behandlung oder signifikanter Rückgang der BMD $\geq 10 \% /$ Jahr nach 1 Jahr Behandlung.
} 
6. Nach Therapie mit Denosumab soll eine Anschlusstherapie mit einem anderen spezifischen Osteoporosemedikament erfolgen.

7. Bei hochgradiger Niereninsuffizienz soll Denosumab eingesetzt werden.

8. Bei Schwangerschaft sollte keine spezifische osteologische Therapie erfolgen.

\section{Diskussion}

Um Hilfestellung in Bezug auf Diagnostik, Risikobeurteilung und Therapie für Patienten mit GIOP in Deutschland zu geben, wurden seitens der DGRh die vorliegenden Behandlungsempfehlungen entwickelt. Sie basieren auf einer systematischen Literatursuche der 2017 aktualisierten ACR-Leitlinie $[19,20]$ mit Erweiterung der Literatursuche um den Zeitraum von 2016 bis 2020, um auch die rezente Evidenz in die Empfehlungen einfließen $z u$ lassen.

Trotz des weit verbreiteten Einsatzes von GC bleibt die GIOP eine unterdiagnostizierte und unterbehandelte Erkrankung. Jüngste Analysen zeigen, dass weniger als $10 \%$ der Patienten, die systemische GC einnehmen (einschließlich derjenigen, die 3 Monate oder länger behandelt werden), einer BMD-Messung zugeführt wurden und lediglich zwischen 12 und $22 \%$ der Patienten unter GC-Langzeittherapie eine knochenprotektive Behandlung erhielten $[57,86]$. Diese Unterversorgung stellt sich bei Männern und jungen Menschen beider Geschlechter noch deutlicher dar. Aus solchen Daten und Erfahrungen, welche den bisherigen Stand als optimierungsbedürftig erscheinen lassen, folgt ein Bedarf an Information und Schulung bezüglich des adäquaten Managements der GIOP. Bisherige nationale und internationale Leitlinien zur GIOP haben Limitationen und divergieren. Nicht speziell auf die GIOP ausgerichtete Empfehlungen bilden meist die mit der GIOP zusammenhängenden komplexen Fragestellungen nicht in vollem Umfang ab. GIOP-Leitlinien unterscheiden sich teilweise in Stratifizierung des Frakturrisikos und insbesondere bezüglich der Indikation für eine pharmakologische Intervention [19, 20, 26, 51, 52, 63, 83].
Einordnung der Empfehlung im Vergleich zu den internationalen Leitlinien

So empfehlen zwar sämtliche GIOP-Leitlinien den Beginn der Therapie unter GC bei einer vorangegangenen Fraktur, allerdings unterscheiden sie sich bezüglich der BMD als Ausgangspunkt für eine Therapieindikation ohne eine Fraktur:

- ACR (USA) und Korean Society for Bone and Mineral Research and the Korean College of Rheumatology (KSBMR-KCR, Korea): T-Score $\leq-2,5[19,20,63]$,

- National Osteoporosis Guideline Group (NOGG, Vereinigtes Königreich): keine Indikation [26],

- Japanese Society for Bone and Mineral Research (JSBMR, Japan): T-Score $\leq-1,5$ [83],

- International Osteoporosis Foundation and European Calcified Tissue Society (IOF-ECTS): behandeln, wenn BMD $<70 \%$ des Mittelwertes der jungen Erwachsenen (20 bis 44 Jahre) [51, 52].

In Abhängigkeit zur GC-Dosis wird die Indikation zur Therapie wie folgt gesehen:

- ACR und KSBMR-KCR: keine Indikation,

- NOGG, JSBMR, IOF-ECTS: behandeln, wenn $\geq 7,5 \mathrm{mg}$ Prednisolonäquivalent.

Und schließlich wird bezüglich des Alters eine knochenprotektive Behandlung wie folgt als sinnvoll bewertet:

- ACR und KSBMR-KCR: keine Indikation,

- NOGG und JSBMR: behandeln $\geq 70$ Jahre,

- IOF-ECTS 2012: behandeln $\geq 70$ Jahre.

In der DVO-Leitlinie (2017) wird der Einsatz osteotroper Medikamente empfohlen bei einer täglichen GC-Dosis von $\geq 7,5 \mathrm{mg}$ Prednisolonäquivalent über 3 Monate ab einem T-Score $\leq 1,5$. Bei einer Dosierung von $\leq 7,5 \mathrm{mg}$ Prednisolonäquivalent müssen zusätzliche Risikofaktoren bei der Festlegung des
Schwellenwertes berücksichtigt werden [1].

Hinsichtlich der internationalen Leitlinien und der deutschsprachigen DVOLeitlinie bestehen keine einheitlichen Kriterien für die Initiierung einer spezifischen osteologischen Medikation bei einer GIOP bzw. im Rahmen des Einsatzes von GC. Ziel der Empfehlung zum Management der GIOP der DGRh ist nicht, die Verifizierung der Therapieschwellen für eine osteologische Therapieindikation $\mathrm{zu}$ definieren. Bezugnehmend auf die oben dargestellten Daten, sollten in einer weiteren wissenschaftlichen Aufarbeitung die Therapieschwellen zur Durchführung einer osteologischen Therapie bei einer GIOP detailliert reevaluiert werden.

Aufgrund dessen, dass der Knochenabbau und das Frakturrisiko umgehend nach Beginn einer GC-Therapie deutlich ansteigen, unterschätzen BMDMessungen und Risikokalkulatoren wie der FRAX-Score vermutlich das Frakturrisiko, v. a. bei Langzeit-GC-Therapien. Darüber hinaus ist der unabhängige Beitrag der Grunderkrankung, die eine GCTherapie erfordert, auf den Knochenstoffwechsel nicht vollständig verstanden und in der klinischen Praxis nicht berücksichtigt. In diesem Zusammenhang ist darzustellen, dass der Knochenmineraldichteverlust schon frühzeitig aufgrund der chronischen Entzündung bei rheumatologischen Erkrankungen (z.B. rheumatoide Arthritis) auftreten kann und durch weitere Faktoren, wie z. B. Alter, weibliches Geschlecht, eine reduzierte Mobilität und einen niedrigen Body-Mass-Index, verstärkt werden kann. Dieser entzündlich bedingte Knochenmineraldichteverlust ist durch die Inhibierung der Entzündung im Rahmen des Einsatzes von GC zu vermindern. Somit haben die GC auch einen potenziell positiven Einfluss auf den Knochenstoffwechsel und die Knochenmineraldichte [64].

Es ist wahrscheinlich, dass entweder das absolute Frakturrisiko oder die Entscheidung, ob eine pharmakologische Intervention angebracht ist, in Abhängigkeit von der zugrunde liegenden entzündlichen Erkrankung sowie der Dosierung und der Dauer der GC-Therapie 
unterschiedlich ausfällt. Gleichzeitig gibt es zu wenig Evidenz, um das Management des Frakturrisikos bei Erwachsenen unter 40 Jahren adäquat zu steuern. Trotz bzw. aufgrund der genannten Einschränkungen empfehlen wir in Ermangelung entsprechender Daten eine Bewertung des Frakturrisikos (z. B. anhand von BMD, Risikokalkulatoren und ggf. der Bewertung von Wirbelfrakturen) für alle Personen, die orale GC für $\geq 3$ Monate erhalten, unabhängig von Alter und Geschlecht. Diese Empfehlung erweitert die der DVO-Leitlinie 2017, welche für Patienten mit einer rheumatoiden Arthritis aufgrund zusätzlicher Risikofaktoren wie einer GC-Exposition eine Basisdiagnostik empfiehlt, um den Aspekt der Langzeit-GC-Therapie. Es werden zukünftig Studien benötigt, um weitere Erkenntnisse für das Osteoporose- bzw. Frakturrisiko und zum Nutzen von Basisdiagnostik einschließlich DXA v. a. von jüngeren $\mathrm{Pa}$ tienten unter Langzeit-GC-Therapie zu gewinnen.

Bei entsprechender Indikation empfehlen wir Bisphosphonate oder Denosumab als Erstlinientherapie. Aufgrund fehlender Daten bezüglich des harten Endpunktes Frakturen wurde keine eindeutige Präferenz angegeben. Aufgrund der Überlegenheit von Denosumab im Vergleich zu Risedronat und auch anderen Bisphosphonaten zumindest bezüglich der besseren BMD-Werte kann der bevorzugte Einsatz von Denosumab in Erwägung gezogen werden.

Bezüglich der Wahl eines Bisphosphonates haben wir uns ähnlich wie bei anderen Empfehlungen, z.B. ACR, da$\mathrm{zu}$ entschieden, keine eindeutige Präferenz innerhalb dieser Gruppe zu empfehlen. Hintergrund ist, dass trotz besserer BMD an der LWS unter Zoledronat gegenüber Risedronat kein signifikanter Unterscheid für die BMD am Femur zu beobachten war und zudem in keiner Studie eine Überlegenheit für Zoledronat bei harten Endpunkten wie Frakturen im Vergleich zu anderen Bisphosphonaten gezeigt wurde.

Trotz Heterogenität der Datenlage sind unsere Empfehlungen an die der DVO-Leitlinie angelehnt.

Ein detailliertes Therapieziel, das bei Verfehlen ggf. zur Umstellung der Thera- pie führt, ist schwierig zu definieren, da bei der GIOP wenig Daten im Sinne eines Treat-to-Target-Ansatzes existieren; zudem unterscheiden sich Therapieziele in Abhängigkeit von Alter, Risikofaktoren, zugrunde liegender entzündlicher Erkrankung und Wirkmechanismus der Osteoporosemedikation ggf. erheblich. Ein mögliches Verfehlen des Therapieziels kann beispielweise angenommen werden, wenn eine neue Fraktur $\geq 18 \mathrm{Mo}$ nate nach Beginn der Behandlung oder ein signifikanter Rückgang der BMD $\geq 10 \% / J a h r$ nach 1 Jahr Behandlung auftritt. Die Risikoeinschätzung muss jedoch immer individuell erfolgen zusammen mit dem Patienten im Sinne einer partizipativen Entscheidungsfindung.

\section{Fazit}

Der Einsatzvon GC ist mit einer Erniedrigung der Knochenmineraldichte und einem erhöhten Frakturrisiko verbunden. Alle Patienten mit einem Einsatz von GC über 3 Monate sollten ein Risikoassessment für eine GIOP und eine Substitution mit Kalzium und Vitamin $\mathrm{D}_{3}$ zur Prophylaxe einer GIOP erhalten. In Abhängigkeit vom Risikoassessment ist eine spezifische osteologische Therapie in Anlehnung an die DVO-Leitlinie (2017) einzuleiten. Bei einer Niereninsuffizienz ist der Einsatz von Denosumab zu präferieren. Nach der Beendigung einer DenosumabTherapie ist ein anderes antiresorptives Therapieverfahren durchzuführen.

\section{Korrespondenzadresse}

\section{Jan Leipe}

Sektion Rheumatologie, Medizinische Klinik V, Universitätskrankenhaus Mannheim, Universitätsklinikum Mannheim

Theodor-Kutzer-Ufer 1-3, 68167 Mannheim, Deutschland

jan.leipe@umm.de

\section{Einhaltung ethischer Richtlinien}

Interessenkonflikt. J. Leipe, J.U. Holle, C. Weseloh, A. Pfeil und K. Krüger geben an, dass kein Interessenkonflikt besteht.
Für diesen Beitrag wurden von den Autoren keine Studien an Menschen oder Tieren durchgeführt. Für die aufgeführten Studien gelten die jeweils dort angegebenen ethischen Richtlinien.

\section{Literatur}

1. AWMF online (2017) Prophylaxe, Diagnostik und Therapie der Osteoporose. https://www. awmf.org/uploads/tx_szleitlinien/183-001I_S3_ Osteoporose-Prophylaxe-Diagnostik-Therapie_ 2019-02.pdf.Zugegriffen:01.07.2021

2. Adachi JD, Bensen WG, Bianchi F et al (1996) Vitamin $D$ and calcium in the prevention of corticosteroid induced osteoporosis: a 3 year followup. JRheumatol 23:995-1000

3. Adachi JD, Saag KG, Delmas PD et al (2001) Twoyear effects of alendronate on bone mineral density and vertebral fracture in patients receiving glucocorticoids: a randomized, double-blind, placebo-controlled extension trial. Arthritis Rheum 44:202-211

4. Adami G, Rahn EJ, Saag KG (2019) Glucocorticoidinduced osteoporosis: from clinical trials to clinical practice. Ther Adv Musculoskelet Dis 11:1759720X19876468

5. Adami G, Saag KG (2019) Glucocorticoid-induced osteoporosis: 2019 concise clinical review. Osteoporos Int 30:1145-1156

6. Adams AL, Adams JL, Raebel MA et al (2018) Bisphosphonate drug holiday and fracture risk: a population-based cohort study. J Bone Miner Res 33:1252-1259

7. Albaum JM, Levesque LE, Gershon AS et al (2015) Glucocorticoid-induced osteoporosis management among seniors, by year, sex, and indication, 1996-2012. Osteoporos Int 26:2845-2852

8. Allen CS, Yeung JH, Vandermeer B et al (2016) Bisphosphonates for steroid-induced osteoporosis. Cochrane Database Syst Rev 10:CD1347

9. Amiche MA, Abtahi S, Driessen JHM et al (2018) Impact of cumulative exposure to high-dose oral glucocorticoids on fracture risk in Denmark: a population-based case-control study. Arch Osteoporos 13:30

10. Amiche MA, Albaum JM, Tadrous $M$ et al (2016) Fracture risk in oral glucocorticoid users: a Bayesian meta-regression leveraging control arms of osteoporosis clinical trials. Osteoporos Int 27:1709-1718

11. Andrews EB, Gilsenan AW, Midkiff $K$ et al (2012) The US postmarketing surveillance study of adult osteosarcoma and teriparatide: study design and findings from the first 7 years. J Bone Miner Res 27:2429-2437

12. Angeli A, Guglielmi G, Dovio A et al (2006) High prevalence of asymptomatic vertebral fractures in post-menopausal women receiving chronic glucocorticoid therapy: a cross-sectional outpatient study. Bone 39:253-259

13. Anonymous (1994) Assessment of fracture risk and its application to screening for postmenopausal osteoporosis. Report of a WHO study group. World Health Organ Tech Rep Ser 843:1-129

14. Awmf (2018) Antiresorptiva-assoziierte Kiefernekrosen. https://www.awmf.org/uploads/ tx_szleitlinien/007-091I_S3_Antiresorptivaassoziierte-Kiefernekrosen-AR-ONJ_2018-12. pdf.Zugegriffen:01.07.2021

15. Balasubramanian A, Wade SW, Adler RA et al (2016) Glucocorticoid exposure and fracture risk 
in patients with new-onset rheumatoid arthritis. Osteoporos Int 27:3239-3249

16. Bolland MJ, Avenell A, Baron JA et al (2010) Effect of calcium supplements on risk of myocardial infarction and cardiovascular events: metaanalysis. BMJ 341:c3691

17. Bolland MJ, Grey A, Avenell A et al (2011) Calcium supplements with or without vitamin D and risk of cardiovascular events: reanalysis of the women's health initiative limited access dataset and metaanalysis. BMJ342:d2040

18. Braun JJ, Birkenhager-Frenkel DH, Rietveld AH etal (1983) Influence of 1 alpha-(OH)D3 administration on bone and bone mineral metabolism in patients on chronic glucocorticoid treatment; a double blind controlled study. Clin Endocrinol (Oxf) 19:265-273

19. Buckley L, Guyatt G, Fink HA et al (2017) 2017 American college of rheumatology guideline for the prevention and treatment of glucocorticoidinduced osteoporosis. Arthritis Care Res (Hoboken) 69:1095-1110

20. Buckley L, Guyatt G, Fink HA et al (2017) 2017 American college of rheumatology guideline for the prevention and treatment of glucocorticoid-induced osteoporosis. Arthritis Rheumato 69:1521-1537

21. Canalis E, Mazziotti G, Giustina A et al (2007) Glucocorticoid-induced osteoporosis: pathophysiology and therapy. Osteoporos Int 18:1319-1328

22. Caplan A, Fett N, Rosenbach M et al (2017) Prevention and management of glucocorticoidinduced side effects: a comprehensive review: a review of glucocorticoid pharmacology and bone health. J Am Acad Dermatol 76:1-9

23. Chen Y, Wan JX, Jiang DW et al (2015) Efficacy of calcitriol in treating glucocorticoidinduced osteoporosis in patients with nephrotic syndrome: an open-label, randomized controlled study. Clin Nephrol 84:262-269

24. Coco M, Pullman J, Cohen HW et al (2012) Effect of risedronate on bone in renal transplant recipients. JAm Soc Nephrol 23:1426-1437

25. Cohen S, Levy RM, Keller Met al (1999) Risedronate therapy prevents corticosteroid-induced bone loss: a twelve-month, multicenter, randomized, double-blind, placebo-controlled, parallel-group study. Arthritis Rheum 42:2309-2318

26. Compston J, Cooper A, Cooper C et al (2017) UK clinical guideline for the prevention and treatment of osteoporosis. Arch Osteoporos 12:43

27. Crawford BA, Kam C, Pavlovic J et al (2006) Zoledronic acid prevents bone loss after liver transplantation: a randomized, double-blind, placebocontrolled trial. Ann Intern Med 144:239-248

28. Cummings SR, Ferrari S, Eastell Ret al (2018) Vertebral fractures after discontinuation of denosumab: a post hoc analysis of the randomized placebocontrolled FREEDOM trial and its extension. JBone Miner Res 33:190-198

29. De Vries F, Bracke M, Leufkens HG et al (2007) Fracture risk with intermittent high-dose oral glucocorticoid therapy. Arthritis Rheum 56:208-214

30. Dore RK, Cohen SB, Lane NE et al (2010) Effects of denosumab on bone mineral density and bone turnover in patients with rheumatoid arthritis receiving concurrent glucocorticoids or bisphosphonates. Ann Rheum Dis 69:872-875

31. Eastell R, Rosen CJ, Black DM et al (2019) Pharmacological management of osteoporosis in postmenopausal women: an endocrine society* clinical practice guideline. J Clin Endocrinol Metab 104:1595-1622
32. European Prospective Osteoporosis Study G, Felsenberg D, Silman AJ et al (2002) Incidence of vertebral fracture in europe: results from the European prospective osteoporosis study (EPOS). JBone Miner Res 17:716-724

33. Feldstein AC, Elmer PJ, Nichols GA et al (2005) Practice patterns in patients at risk for glucocorticoid-induced osteoporosis. Osteoporos Int 16:2168-2174

34. Fink HA, Macdonald R, Forte ML et al (2019) Long term drug therapy and drug discontinuations and holidays for osteoporosis fracture prevention: a systematic review. Ann Intern Med 171:37-50

35. Gluer CC, Marin F, Ringe JD et al (2013) Comparative effects of teriparatide and risedronate in glucocorticoid-induced osteoporosis in men: 18month results of the EuroGIOPs trial. J Bone Miner Res 28:1355-1368

36. Gu C, Zhao R, Zhang X et al (2019) A metaanalysis of secondary osteoporosis in systemic lupus erythematosus: prevalence and risk factors. Arch Osteoporos 15:1

37. Guadalix S, Martinez-Diaz-Guerra G, Lora D et al (2011) Effect of early risedronate treatment on bone mineral density and bone turnover markers after liver transplantation: a prospective singlecenter study. Transpl Int 24:657-665

38. Hakala M, Kroger H, Valleala H et al (2012) Oncemonthly oral ibandronate provides significant improvement in bone mineral density in postmenopausal women treated with glucocorticoids fo inflammatory rheumatic diseases: a 12-month, randomized, double-blind, placebo-controlled trial. Scand J Rheumatol 41:260-266

39. Hall GM, SpectorTD, Griffin A J et al (1993) The effect of rheumatoid arthritis and steroid therapy on bone density in postmenopausal women. Arthritis Rheum 36:1510-1516

40. Hsia J, Heiss G, Ren H et al (2007) Calcium/vitamin D supplementation and cardiovascular events. Circulation 115:846-854

41. Icks A, Haastert B, Wildner M et al (2008) Trend of hip fracture incidence in Germany 1995-2004: a population-based study. Osteoporos Int 19:1139-1145

42. Iseri K, lyoda M, Watanabe Met al (2018) The effects of denosumab and alendronate on glucocorticoidinduced osteoporosis in patients with glomerular disease: a randomized, controlled trial. PLOS ONE 13:e193846

43. Jackson RD, Lacroix AZ, Gass M et al (2006) Calcium plus vitamin $D$ supplementation and the risk of fractures. NEngl J Med 354:669-683

44. Kanis JA, Johansson $H$, Oden A et al (2011) Guidance for the adjustment of FRAX according to the dose of glucocorticoids. Osteoporos Int 22:809-816

45. Karras D, Stoykov I, Lems WF et al (2012) Effectiveness of teriparatide in postmenopausal women with osteoporosis and glucocorticoid use 3-year results from the EFOS study. J Rheumatol 39:600-609

46. Khan B, Nowson CA, Daly RM et al (2015) Higher dietary calcium intakes are associated with reduced risks of fractures, cardiovascular events, and mortality: a prospective cohort study of olde men and women. JBone Miner Res 30:1758-1766

47. Kidney Disease: Improving Global Outcomes (KDIGO) CKD-MBD Update Work Group (2011) KDIGO 2017 clinical practice guideline update for the diagnosis, evaluation, prevention, and treatment of chronic kidney disease-mineral and bone disorder (CKD-MBD). Kidney Int Suppl 7:1-59
48. Laan RF, van Riel PL, van de Putte LB et al (1993) Low-dose prednisone induces rapid reversible axial bone loss in patients with rheumatoid arthritis. A randomized, controlled study. Ann Intern Med 119:963-968

49. Lane NE, Sanchez S, Modin GW et al (1998) Parathyroid hormone treatment can reverse corticosteroid-induced osteoporosis. Results of a randomized controlled clinical trial. J Clin Invest 102:1627-1633

50. Langdahl BL, Silverman S, Fujiwara $S$ et al (2018) Real-world effectiveness of teriparatide on fracture reduction in patients with osteoporosis and comorbidities or risk factors for fractures: Integrated analysis of 4 prospective observational studies. Bone 116:58-66

51. Lekamwasam S, Adachi JD, Agnusdei D et al (2012) A framework for the development of guidelines for the management of glucocorticoid-induced osteoporosis. Osteoporos Int 23:2257-2276

52. Lekamwasam S, Adachi JD, Agnusdei D et al (2012) An appendix to the 2012 IOF-ECTS guidelines for the management of glucocorticoid-induced osteoporosis. Arch Osteoporos 7:25-30

53. Lems WF, Lodder MC, Lips P et al (2006) Positive effect of alendronate on bone mineral density and markers of bone turnover in patients with rheumatoid arthritis on chronic treatment with low-dose prednisone: a randomized, doubleblind, placebo-controlled trial. Osteoporos Int 17:716-723

54. Leslie WD, Adler RA, El-Hajj Fuleihan G et al (2006) Application of the 1994 WHO classification to populations other than postmenopausal Caucasian women: the 2005 ISCD official positions. JClin Densitom 9:22-30

55. Li K, Kaaks R, Linseisen J et al (2012) Associations of dietary calcium intake and calcium supplementation with myocardial infarction and stroke risk and overall cardiovascular mortality in the Heidelberg cohort of the European prospective investigation into cancer and nutrition study (EPIC-Heidelberg). Heart 98:920-925

56. Locascio V, Bonucci E, Imbimbo B et al (1990) Bone loss in response to long-term glucocorticoid therapy. Bone Miner 8:39-51

57. Majumdar SR, Lix LM, Morin SN et al (2013) The disconnect between better quality of glucocorticoid-induced osteoporosis preventive care and better outcomes: a population-based cohort study. JRheumatol 40:1736-1741

58. Majumdar SR, Lix LM, Yogendran $M$ et al (2012) Population-based trends in osteoporosis management after new initiations of long-term systemic glucocorticoids (1998-2008). J Clin Endocrinol Metab 97:1236-1242

59. Mcdonough AK, Curtis JR, Saag KG (2008) The epidemiology of glucocorticoid-associated adverse events. Curr Opin Rheumatol 20:131-137

60. Michos ED, Blumenthal RS (2007) Vitamin D supplementation and cardiovascular disease risk. Circulation 115:827-828

61. Mok CC, Ho LY, Ma KM (2015) Switching of oral bisphosphonates to denosumab in chronic glucocorticoid users: a 12-month randomized controlled trial. Bone 75:222-228

62. Okada Y, Nawata M, Nakayamada S et al (2008) Alendronate protects premenopausal women from bone loss and fracture associated with high-dose glucocorticoid therapy. J Rheumato 35:2249-2254

63. Park SY, Gong HS, Kim KM et al (2018) Korean guideline for the prevention and treatment 
of glucocorticoid-induced osteoporosis. J Bone Metab 25:195-211

64. Raterman HG, Bultink IE, Lems WF (2020) Osteoporosis in patients with rheumatoid arthritis: an update in epidemiology, pathogenesis, and fracture prevention. Expert Opin Pharmacother 21:1725-1737

65. Reid DM, Devogelaer JP, Saag K et al (2009) Zoledronic acid and risedronate in the prevention and treatment of glucocorticoid-induced osteoporosis (HORIZON): a multicentre, double-blind, double-dummy, randomised controlled trial. Lancet 373:1253-1263

66. Reid DM, Hughes RA, Laan RF et al (2000) Efficacy and safety of daily risedronate in the treatment of corticosteroid-induced osteoporosis in men and women: a randomized trial. European corticosteroid-induced osteoporosis treatment study. JBone Miner Res 15:1006-1013

67. Roux C, Briot K (2017) Imminent fracture risk. Osteoporos Int 28:1765-1769

68. Saag KG, Agnusdei D, Hans D et al (2016) Trabecular bone score in patients with chronic glucocorticoid therapy-induced osteoporosis treated with alendronate or teriparatide. Arthritis Rheumatol 68:2122-2128

69. Saag KG, Emkey R, Schnitzer TJ et al (1998) Alendronate for the prevention and treatment of glucocorticoid-induced osteoporosis. Glucocorticoid-induced osteoporosis intervention study group. NEngl J Med 339:292-299

70. Saag KG, Koehnke R, Caldwell JR et al (1994) Low dose long-term corticosteroid therapy in rheumatoid arthritis: an analysis of serious adverse events. Am J Med 96:115-123

71. Saag KG, Pannacciulli N, Geusens P et al (2019) Denosumab versus risedronate in glucocorticoidinduced osteoporosis: final results of a twentyfour-month randomized, double-blind, doubledummy trial. Arthritis Rheumatol 71:1174-1184

72. Saag KG, Shane E, Boonen S et al (2007) Teriparatide or alendronate in glucocorticoid-induced osteoporosis. NEngl J Med 357:2028-2039

73. Saag KG, Wagman RB, Geusens P et al (2018) Denosumab versus risedronate in glucocorticoidinduced osteoporosis: a multicentre, randomised, double-blind, active-controlled, double-dummy, non-inferiority study. Lancet Diabetes Endocrinol 6:445-454

74. Saag KG, Zanchetta JR, Devogelaer JP et al (2009) Effects of teriparatide versus alendronate for treating glucocorticoid-induced osteoporosis: thirtysix-month results of a randomized, double-blind, controlled trial. Arthritis Rheum 60:3346-3355

75. Salerno A, Hermann R (2006) Efficacy and safety of steroid use for postoperative pain relief. Update and review of the medical literature. J Bone Joint Surg Am 88:1361-1372

76. Sambrook P, Birmingham J, Kelly P et al (1993) Prevention of corticosteroid osteoporosis. A comparison of calcium, calcitriol, and calcitonin. NEngl J Med 328:1747-1752

77. Sambrook PN, Roux C, Devogelaer JP et al (2012) Bisphosphonates and glucocorticoid osteoporosis in men: results of a randomized controlled trial comparing zoledronic acid with risedronate. Bone 50:289-295

78. Shane E, Cohen A, Stein EM et al (2012) Zoledronic acid versus alendronate for the prevention of bone loss after heart or liver transplantation. J Clin Endocrinol Metab 97:4481-4490

79. Shin K, Park SH, Park W et al (2017) Monthly oral ibandronate reduces bone loss in Korean women with rheumatoid arthritis and osteopenia receiving long-term glucocorticoids: a 48-week double-blinded randomized placebo-controlled investigator-initiated trial.Clin Ther39:268-278.e2

80. Solomon DH, Katz JN, Jacobs JP et al (2002) Management of glucocorticoid-induced osteoporosis in patients with rheumatoid arthritis: rates and predictors of care in an academic rheumatology practice. Arthritis Rheum 46:3136-3142

81. Steinbuch M, Youket TE, Cohen S (2004) Oral glucocorticoid use is associated with an increased risk of fracture. Osteoporos Int 15:323-328

82. Stoch SA, Saag KG, Greenwald M et al (2009) Once-weekly oral alendronate $70 \mathrm{mg}$ in patients with glucocorticoid-induced bone loss: a 12month randomized, placebo-controlled clinical trial. JRheumatol 36:1705-1714

83. Suzuki Y, Nawata H, Soen S et al (2014) Guidelines on the management and treatment of glucocorticoid-induced osteoporosis of the Japanese society for bone and mineral research: 2014 update. JBone Miner Metab 32:337-350

84. Tee SI, Yosipovitch G, Chan YC et al (2012) Prevention of glucocorticoid-induced osteoporosis in immunobullous diseases with alendronate: a randomized, double-blind, placebo-controlled study. Arch Dermatol 148:307-314

85. Torregrosa JV, Fuster D, Gentil MA et al (2010) Open-label trial: effect of weekly risedronate immediately after transplantation in kidney recipients. Transplantation 89:1476-1481

86. Trijau S, de Lamotte G, Pradel V et al (2016) Osteoporosis prevention among chronic glucocorticoid users: results from a public health insurance database. RMD Open 2:e249

87. Van Hemelrijck M, Michaelsson K, Linseisen J et al (2013) Calcium intake and serum concentration in relation to risk of cardiovascular death in NHANES III.PLoSOne 8:e61037

88. Van Staa TP, Laan RF, Barton IP et al (2003) Bone density threshold and other predictors of vertebral fracture in patients receiving oral glucocorticoid therapy. Arthritis Rheum 48:3224-3229

89. van Staa TP, Leufkens HG, Abenhaim L et al (2000) Oral corticosteroids and fracture risk: relationship to daily and cumulative doses. Rheumatology (Oxford) 39:1383-1389

90. Van Staa TP, Leufkens HG, Abenhaim L et al (2000) Use of oral corticosteroids and risk of fractures. JBone Miner Res 15:993-1000

91. van Staa TP, Leufkens HG, Cooper C (2002) The epidemiology of corticosteroid-induced osteoporosis: a meta-analysis. Osteoporos Int 13:777-787

92. Wallach S, Cohen S, Reid DM et al (2000) Effects of risedronate treatment on bone density and vertebral fracture in patients on corticosteroid therapy. CalcifTissue Int 67:277-285

93. Xiao Q, Murphy RA, Houston DK et al (2013) Dietary and supplemental calcium intake and cardiovascular disease mortality: the national institutes of health-AARP diet and health study. JAMA Intern Med 173:639-646

94. Yamada S, Takagi $H$, Tsuchiya $H$ et al (2007) Comparative studies on effect of risedronate and alfacalcidol against glucocorticoid-induced osteoporosis in rheumatoid arthritic patients. Yakugaku Zasshi 127:1491-1496

95. Yanbeiy ZA, Hansen KE (2019) Denosumab in the treatment of glucocorticoid-induced osteoporosis: a systematic review and meta-analysis. Drug Des Devel Ther 13:2843-2852
Risikofaktoren für schwere COVID-19-Verläufe bei Rheumapatienten

Erste Daten aus dem COVID-19-Register der DGRh definieren Risikofaktoren für schwere Verläufe, die mit einer rheumatischen Grunderkrankung zusammenhängen.

Sind Rheumapatienten bei einer SARSCoV-2-Infektion besonderen Risiken ausgesetzt und welchen Einfluss hat die Rheumamedikation? Um diese Fragen zu klären, hat die Deutsche Gesellschaft für Rheumatologie e. V. (DGRh) gemeinsam mit der Universität Gießen das Online-Register "Covid19-rheuma.de" ins Leben gerufen. In diesem werden Covid-19-Krankheitsverläufe von Patienten mit Rheuma dokumentiert.

Dabei bestätigen sich zunächst die auch für die Allgemeinbevölkerung geltenden Erkenntnisse zu SARS-CoV-2: Als unabhängiger Risikofaktor für einen schweren Verlauf zeigte sich besonders das Alter über 65-Jährige hatten ein 2,24-mal, über 75-Jährige sogar ein fast 4-mal so hohes Hospitalisierungsrisiko wie jüngere Patienten. Auch die Art und Anzahl der zusätzlichen Begleiterkrankungen beeinflusste den Verlauf der Erkrankung deutlich.

Daneben lassen sich aber auch Risikofaktoren ausmachen, die speziell mit einer rheumatischen Grunderkrankung und ihrer Therapie in Verbindung stehen: Als Risikogruppe erwiesen sich insbesondere Patienten, die täglich mehr als $5 \mathrm{mg}$ Glukokortikoide einnahmen, sowie solche, deren aktuelle Krankheitsaktivität als moderat bis hoch eingeschätzt wurde.

Während der Pandemie sollte daher auf eine möglichst gute medikamentöse Kontrolle der rheumatischen Grunderkrankung geachtet werden und, wo immer möglich, auf die dauerhafte Gabe höher dosierter Glukokortikoide verzichtet werden.

Quelle: Deutsche Gesellschaft für Rheumatologie e. V., www.dgrh.de 